\title{
Simultaneous Synthesis of Heat Exchanger Networks with Operability Considerations: Flexibility and Controllability
}

\author{
Marcelo Escobar ${ }^{*}$, Jorge O. Trierweiler \\ Chemical Engineering Department, Federal University of Rio Grande do Sul (UFRGS)-Brazil

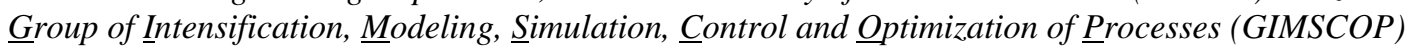 \\ e-mail: escobar@enq.ufrgs.br/jorge@enq.ufrgs.br
}

Ignacio E. Grossmann

Chemical Engineering Department, Carnegie Mellon University (CMU)- USA

Center of Advanced Process Decision Making (CAPD)

e-mail: grossmann@cmu.edu

\section{ABSTRACT}

In this work a computational framework is proposed for the synthesis of flexible and controllable Heat Exchanger Networks. The synthesis is projected to operate over a specified range of expected variations in the inlet temperatures and flowrates of the process streams using a decentralized control system, such that the Total Annual Cost involving the utility consumption and the investment are optimized simultaneously. The framework is based on a two-stage strategy. A design stage is performed prior to the operability analysis where the design variables are chosen. In the second stage, the control variables are adjusted during operation on the realizations of the uncertain parameters. The framework yields a HEN design, which is guaranteed to operate with the designed control system under varying conditions ensuring stream temperature targets and optimal energy integration. The application of the proposed framework and its computational efficiency are illustrated with some numerical examples.

Keywords: heat integration, heat exchanger network synthesis, operability, flexibility, controllability.

\footnotetext{
${ }^{*}$ Corresponding author. Tel.: +55-51-3308-4072; fax: +55-51-3308-3277.

E-mail: escobar@enq.ufrgs.br
} 


\section{Introduction}

Heat Exchanger Network Synthesis (HENS) is an important part in the overall chemical process. From the energy point of view, an overall process system can be viewed as three main interactive components (see Figure 11) that can be integrated into an operable plant (Aaltola, 2002).

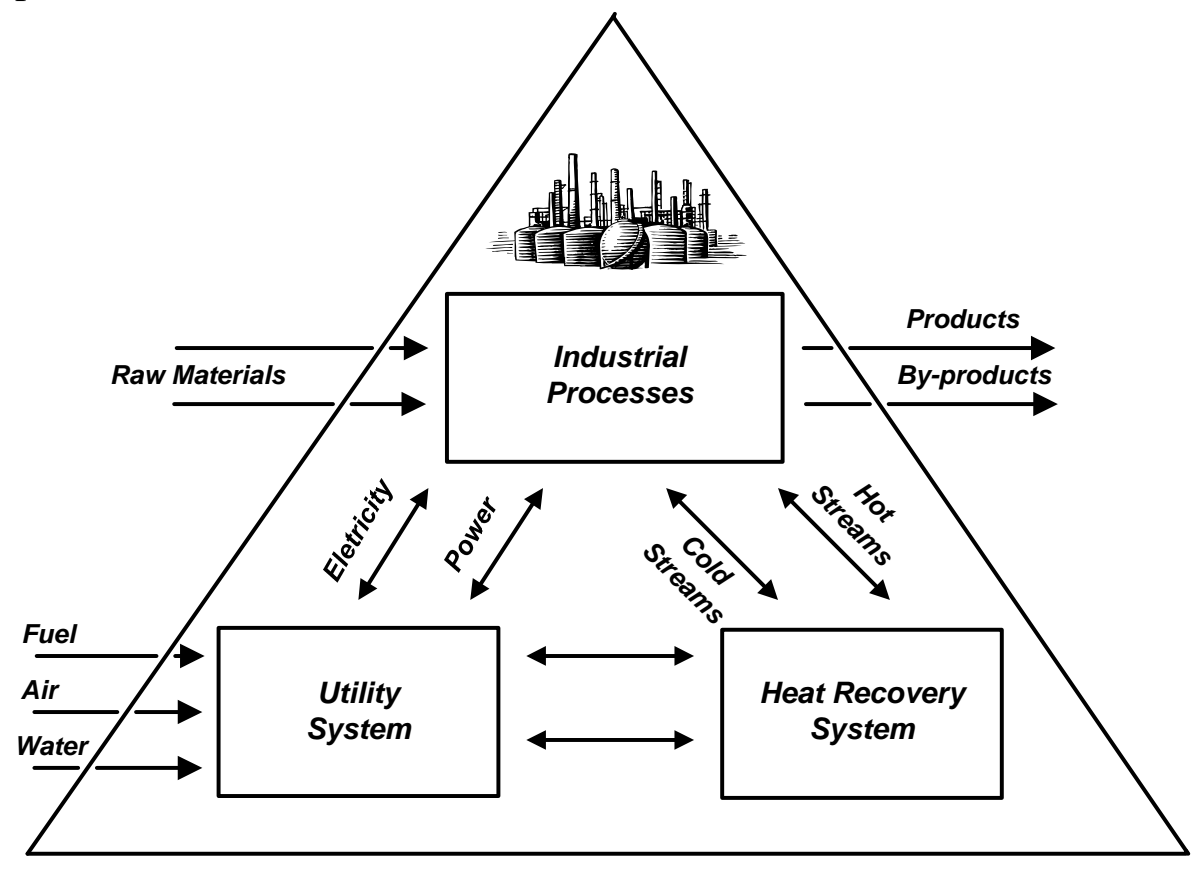

Figure 1. An overall process system.

HENS links the process flowsheet with the utility system and generally involves a large fraction of both the overall plant capital cost, and operating costs in terms of energy requirements, which is a key factor for a profitable process (Verheyen and Zhang, 2006). The aim of the synthesis consists of finding a network design that minimizes the total annualized cost, i.e. the investment cost in units and the operating cost in terms of utility consumption. The major techniques are based on sequential approaches (Linnhoff and Hindmarsh, 1983; Papoulias and Grossmann, 1983; Ciric, Floudas and Grossmann, 1986) and simultaneous approaches (Ciric and Floudas, 1991; Yee and Grossmann, 1990). For a recent review, see the paper by Furman and Sahinidis (2002), and for a case study comparison see Escobar and Trierweiler (2013). In spite of its complexity, the main advantage of simultaneous approaches is that they can handle explicitly all the trade-offs between capital and operating costs. 
The simultaneous approach of Yee and Grossmann (1990) (SYNHEAT model) features interesting characteristics such as being easy to implement and to solve since the feasible region is defined by linear constraints, making this model very attractive for extensions and further considerations. In fact, different extensions have been proposed in the literature such as: (i) removal of the isothermal mixing assumption: Björk and Westerlund (2002); (ii) retrofit: Ma, Hui and Yee (2000); (iii) detailed equipment design and fluid dynamic considerations as pressure drop: Mizutani, Pessoa, Queiroz, Hauan, and Grossmann (2003), Serna-González, Ponce-Ortega, and Jiménez-Gutiérrez (2004); Ravagnani and Caballero (2007); (iv) occurrence of phase change: Ponce-Ortega, Jiménez-Gutiérrez and Grossmann (2008). (v) flexibility considerations: Konukman, Çamurdan, and Akman (2002); Chen and Hung (2004); and Verheyen and Zhang (2006); (vi) environment impacts: López-Maldonado, Ponce-Ortega, and SegoviaHernández (2011).

The conventional Heat Exchanger Network Synthesis is performed under the assumption of fixed operating parameters at nominal conditions. However, it is possible that there are significant changes (uncertainties) in the environment of a plant (Verheyen and Zhang, 2006). Uncertainty is an inherent characteristic of any process system. Therefore, it is important that the design has the ability to maintain feasible steady-state operation under uncertain conditions. In the previous works on flexibility, it is usually assumed that a simple control system can be designed in order to operate the network, and keep the process operating in the level for which the synthesis was optimized.

Although energy integration is motivated by economic benefits, the network configuration impacts the process behavior introducing interactions, and in many cases, making the process more difficult to control and operate (Mathisen, 1994). In addition, the HEN configuration may impose control limitations, such as competitive effects, inverse response, time delay and interaction. These limitations associated with disturbance propagation through the network may make the control extremely difficult, if the network is improperly designed. 
When designing a HEN it is important to have a strategy for operation and control that results in reasonable dynamic performance of the plant. In this context, the term controllability usually means in simple terms how easy the process (network) is to control. Controllability metrics, usually assessed by open loop indicators and linear transfer function models, aim to find what control performance can be expected, or whether a specific closed loop performance can be achieved. While flexibility is concerned with steady-state properties, controllability is related to short-term responses and dynamic properties.

The term operability is often referred to the ease with which a process can be operated and controlled. It includes both flexibility and controllability, and it is strongly affected by the network design. It is important to have in mind that a good HEN design must not only exhibit an optimal trade-off between capital and operating costs, it must also exhibit operability characteristics that will allow this economic performance to be achievable in a practical operating environment. Operability considerations, such as flexibility and controllability, are very important in a HEN design, but they are usually neglected, especially the controllability, during the design phase for a given steady-state operating point (Glemmstad, 1997).

The work of Marselle et al. (1982) was a pioneer in operability considerations for HENs. It was proposed a manual combination of a series of optimal designs under different worst-case scenarios. Kotjabasakis and Linnhoff (1986) introduced the sensitivity tables for the design of flexible HENs. To measure the degree of flexibility of a HEN, Saboo et al. (1985) proposed the Resilience Index (RI), and Swaney and Grossmann (1985) introduced a Flexibility Index (FI). Grossmann and Floudas (1987) introduced an active set strategy for the calculation of the FI. Multiperiod/multiscenario formulations were proposed by Floudas and Grossmann (1987) based on the sequential approach for HENs combined with active set strategy to design flexible HENs. Papalexandri and Pistikopoulos (1994) formulated a large and complex MINLP multiperiod problem for the synthesis and retrofit of flexible and structurally controllable HENs. 
Some authors have focused on the optimal operation and the control strategy for a given HEN structure. For instance, Mathisen (1994) and Glemmestad (1997) extensively studied the control and the optimal operation of HENs, respectively. Uzturk and Akman (1997) described a method for optimal retrofit design of a HEN using bypasses and centralized and decentralized structures. Aguilera and Marchetti (1998) proposed a method for on-line optimization and control of HENs, solving an LP for the optimal operation. Yan et al. (2001) developed a bypass design for controllable Heat Exchanger Networks. Gonzales et al. (2006) proposed a two-level control structure where the high level is a supervisory online optimizer for a model predictive control (MPC). Lersbamrungsuk et al. (2008) proposed a systematic procedure to find a control structure design for optimal operation of HENs, solving an integer problem to define the best pairs for split-range and selective control.

Most recent works have developed multiperiod formulations based on SYNHEAT model of Yee and Grossmann (1990) for synthesis of flexible HENs. Particularly, Aaltola (2002), Chen and Hung (2004), and Verheyen and Zhang (2006).

The optimal design under uncertainty for a general process was discussed in the work of Rooney and Biegler (2003). In their work the uncertainty was separated into two groups, denoting parametric uncertainty (e.g. model parameters) and process variability (e.g. flow rates, process conditions, and inputs). Both are not well known at the design stage, but the later are specified deterministically or measure accurately at some later operating stage and therefore, control (manipulated) variables can be adjusted to compensate this variability.

In these previous works on flexible designs it is assumed that all control variables can be adjusted during the operation. However, the strategy for control and operation is neglected. The procedure proposed here can be seen as an extension of the model of Rooney and Biegler (2003) applied for HENS, in which the control strategy is explicitly considered at the operating stage. In this context, controllability metrics are used as an auxiliary tool to select a suitable control strategy. 
In this work we investigate the incorporation of flexibility and controllability, in the synthesis stage using the SYNHEAT model as a basis. We present a computational framework based on a two-stage strategy, coupling a design stage with an operability analysis for addressing the problem of optimal synthesis in terms of costs, in which flexibility and control design considerations are simultaneously accomplished. The remainder of the paper is organized as follows. Section 2 provides the problem statement to be addressed in this work, and also general ideas about operation and control of HENs are given as background. The mathematical formulation for each problem involved in the proposed framework and the outline of the solution strategy are presented in section 3. In section 4, three examples are presented to illustrate the proposed approach. Finally, conclusions and final remarks are drawn in section 5.

\section{Background}

\subsection{Problem statement}

The problem to be addressed in this paper can be stated as follows. Given are: (i) the stream data; (ii) a specified range for the uncertainties, i.e. inlet temperatures and heat capacity flowrates, for which the flexibility of the network is desired (flexibility target); and (iii) a minimum temperature approach $\left(\Delta T_{\min }\right)$; The problem consists in synthesizing a heat exchanger network with minimum Total Annual Cost (operating and capital investment cost) that is able to operate feasibly under the specified uncertainty/disturbance range and it is possible to operate using a simple decentralized feedback control system.

The following general assumptions are related to this work: (i) constant physical properties; (ii) non occurrence of phase change; (iii) counter-current heat exchanger; (iv) pressure drop and further fluid dynamics considerations are neglected; ( $v$ ) bypasses can be placed across all heat exchangers; (vi) only utility duties and bypasses can be adjusted during operation (potential manipulations); (vii) perfect control during the flexibility analysis, i.e. control can be adjusted to compensate uncertain parameters and no delays in the measurements, or adjustments in the control variables are considered; 
(viii) a decentralized control structure with PI controllers, since it is the most common strategy in industrial processes; (ix) outlet temperatures as controlled variables; (ix) inlet temperatures and heat capacity flow are uncertainties, addressed as disturbances. Additional considerations are explicitly made afterwards in the text.

\subsection{Operation and Control of HENs}

Traditional methods for HENS aim to design a network that yields a reasonable tradeoff between capital and operating cost. A prerequisite for optimal operation is that the HEN is sufficient flexible, i.e. it has the ability to operate over a range of uncertain conditions (disturbances), while satisfying the performance specifications at steadystate. In order to ensure that target temperatures of all hot and cold streams are attained during the network operation, a regulatory control system must be implemented. The main control objective in a HEN is usually to maintain the outlet temperatures to specified target values (setpoints, references). In some case, there may be other control objectives, such as the heat duty of the reboiler or condenser in a distillation column that may be integrated in the HEN, or even some internal temperature that has a maximum value, e.g. to prevent decomposition. Furthermore, there may be outlet temperatures without any specified target value (free outlet temperatures) . In addition to the regulatory control objective, it is also important that the utility cost is as low as possible.

For a fixed design a HEN is considered to be optimally operated if the targets temperatures are satisfied at steady state (main objective), the utility cost is minimized (secondary goal), and the dynamic behavior is satisfactory (Glemmestad, 1997). During the operation, degrees of freedom or manipulated inputs are needed for regulatory control and optimization. The most common possibilities are sketched in Figure 2 and consist of: 1-Utility Flowrates; 2-Bypass fraction; 3-Split fraction; 4-Process Streams flowrates; 5-Exchanger area (e.g. flooded condenser); 6-Recycle (e.g. if exchanger fouling is reduced by increased flowrates). In a decentralized control system, each controlled variable is paired with one manipulation, resulting in a control loop. The whole set of loops constitutes the control structure. For this purpose, the first three 
options are the most generally used. While split fractions may result in competitive effects and possible inverse response, which limit the control performance, only the first two options are addressed in this work. In addition, split fractions are often used for optimization purposes rather than for regulatory control.

It is rather evident when a utility exchanger is the last exchanger for each stream, the manipulation of its utility duty to control the outlet temperature of the stream fulfills the main pairing rule, i.e. provide a fast and direct effect, with no interaction with other control loops. However, if the heat is well integrated not all streams have a utility exchanger, and some bypasses must be allocated to the control structure. The selection of suitable sets of manipulated variables for disturbance rejection is a challenging problem because of its combinatorial nature. The controllability is strongly dependent on both the network configuration and control structure selection.

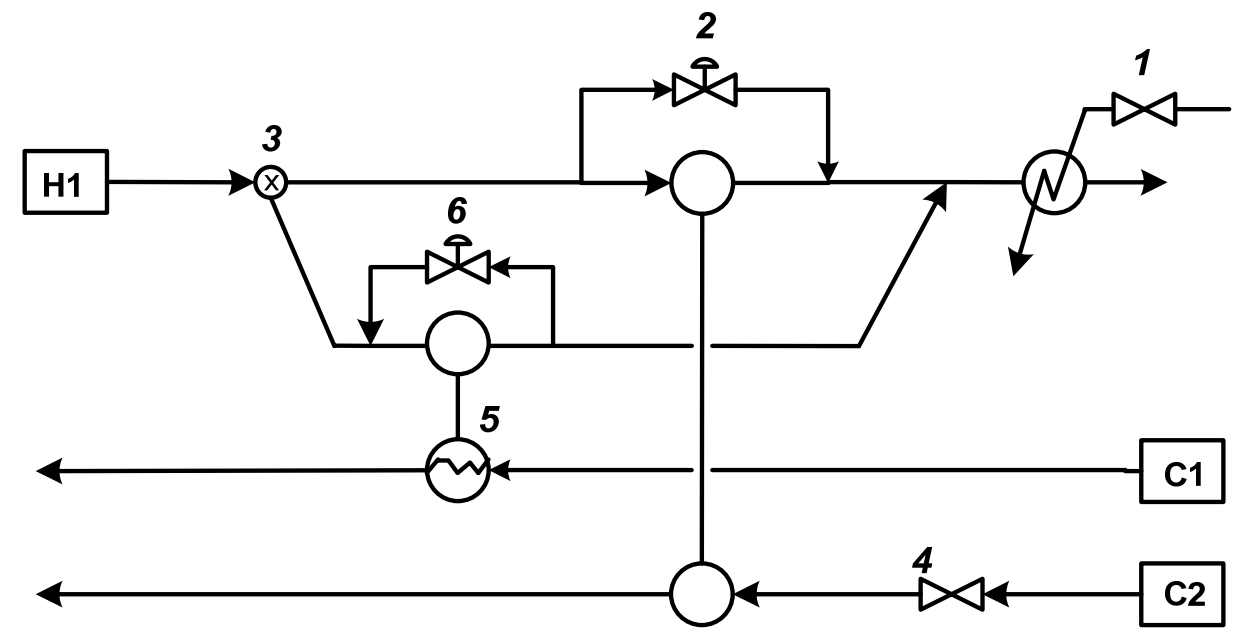

Figure 2. Possible manipulated inputs in HENs.

\subsection{Outline of the proposed strategy}

The incorporation of uncertainty into design may be possible through a deterministic approach based on the postulation of a finite number of periods (scenarios) to characterize the uncertainty and the problem can be formulated as multiscenario/multiperiod optimization problem. The solution of the multiperiod design problem can be embedded in a two-stage strategy (see Figure 3) in order to generate flexible heat exchanger networks (Halemane and Grossmann, 1983; Biegler et al., 1997; 
Aaltola, 2002; Chen and Hung, 2004, 2007). The first stage is prior to the operation (design phase) where the design variables are chosen. At the second stage the control variables are adjusted during operation on the realizations of the uncertain parameters. In the work of Escobar et al. (2011) a computational framework was presented (SYNFLEX) based on this two-stage strategy oriented to the SYNHEAT model for the automatic generation of flexible designs.

Once the design is flexible, in order to operate the network, a control system strategy must be implemented. Different authors have considered the control system design for HENs in the literature (Mathisen, 1994; Aguilera and Marchetii, 1998; Yan et al., 2001; Lersbamrungsuk et al., 2008). It is important to point out that depending on the control strategy the full flexibility cannot be achieved. The actual flexibility may be limited by the control strategy. While the flexibility is a property of the HEN design, the effective flexibility is a property of the HEN design and the control system strategy. In general, only with advanced control techniques and/or online optimization is possible to achieve full flexibility. However, it is also interesting whenever possible to implement a simple decentralized control strategy using low order controllers, e.g. PID controllers. Such control systems are easier to understand and to implement in operation, and also more tolerant to failures than general multivariable control systems. There are classical methods based on a controllability analysis for designing such control structures for a given process, which can be embedded in a modified two-stage strategy proposed here and depicted in Figure 4. For this case, the effective flexibility, i.e. the flexibility of the controlled HEN, i.e. after the control structure implementation, must be evaluated for the operability analysis. In the next section each step and its respective mathematical formulation is presented. 


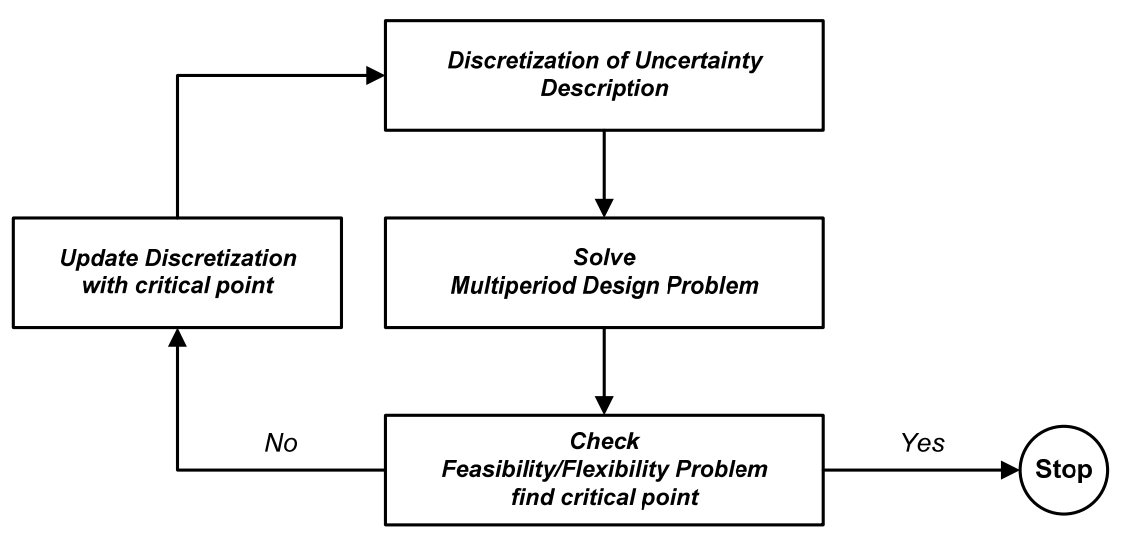

Figure 3. Two-stage strategy for optimal design under uncertainty.

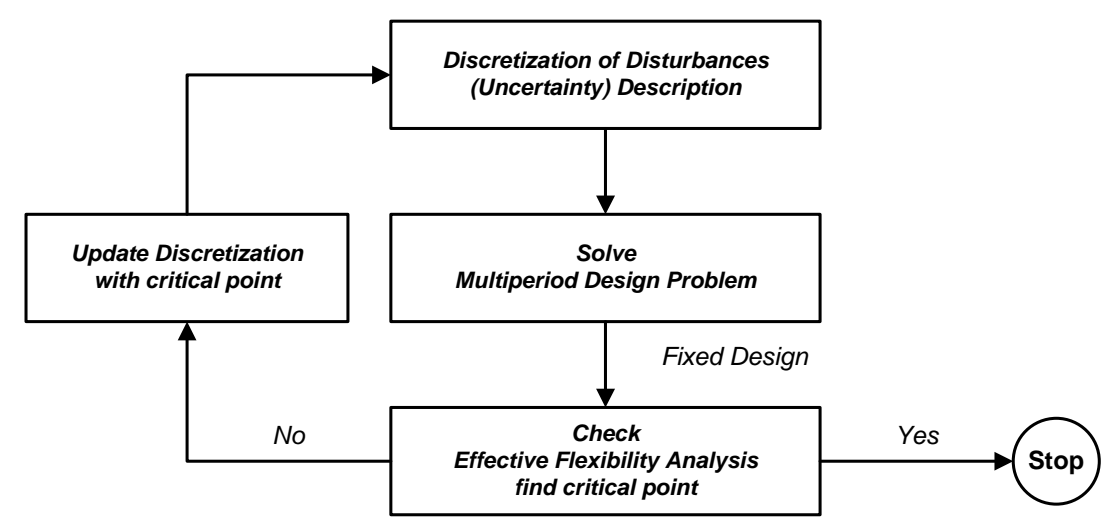

Figure 4. Outline of the proposed strategy for flexible and controllable design.

\section{Mathematical Formulation}

\subsection{Multiperiod Synthesis of Heat Exchanger Networks}

In this work, the design model is based on the stage-wise superstructure proposed by Yee and Grossmann (1990) (SYNHEAT model) depicted in Figure 5. The aim of the model is to find a network configuration that minimizes the total annualized cost, i.e. the investment cost in units and the operating cost in terms of utility consumption. Different multiperiod formulations based on the SYNHEAT model have been proposed in the literature in the last years. In this particular work we consider the formulation proposed by Verheyen and Zhang (2006) and also used in Cheng and Hung (2007). This model features the same assumptions as the SYNHEAT model. However, some nonlinear inequalities are enforced in order to ensure that the installed area, used to calculate the investment cost, is the maximum required area over all periods. 


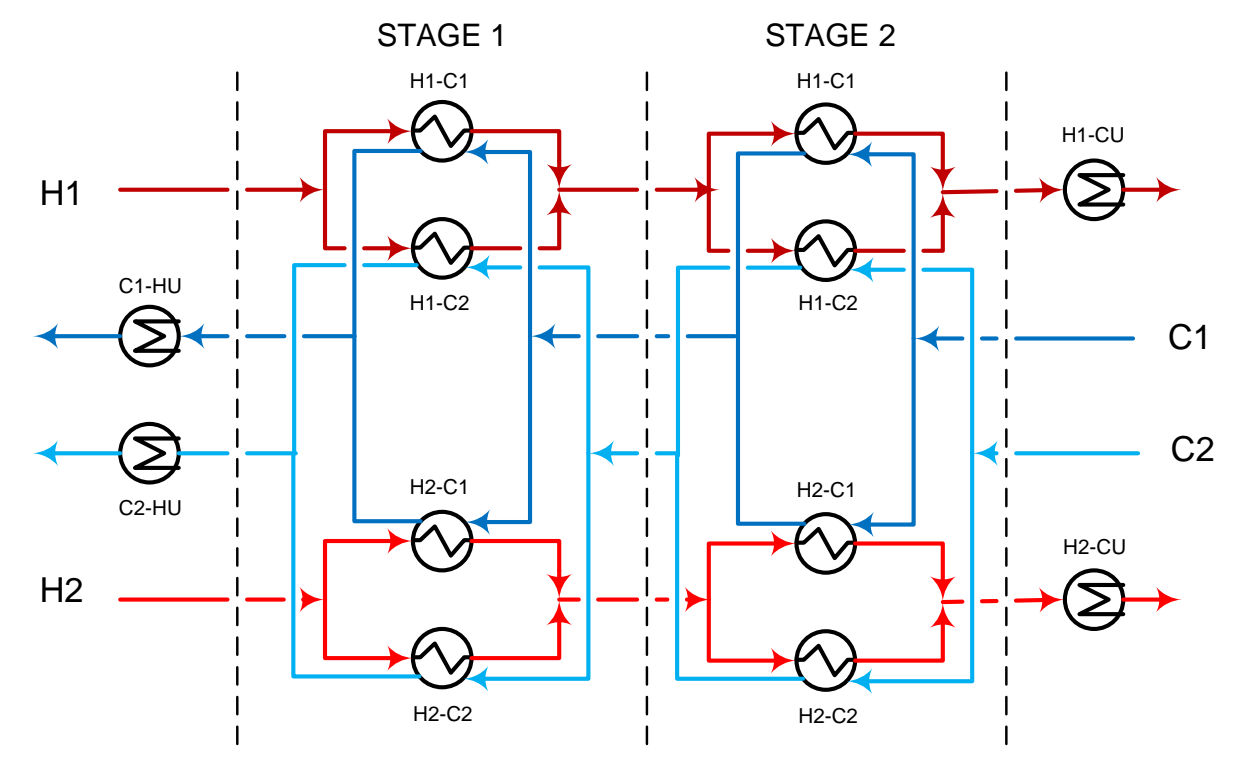

Figure 5. Superstructure for SYNHEAT model (Yee and Grossman, 1990) exemplified for two hot streams, two cold streams and two stages.

The complete model is shown in Appendix A. In the model formulation, an overall heat balance for each period is needed to ensure sufficient heating or cooling of each process stream for each period. As in the SYNHEAT model, it is assumed that the outlet streams of each heat exchanger are mixed isothermically at each stage. For that special case, the energy balances around the mixers are no longer needed. For each hot and cold stream the outlet temperatures leaving the heat exchangers at the same stage $k$ are to be the same, and they are associated with the downstream temperature location $\left(t_{i k+1}\right.$ for hot stream $i$ and $t_{j k}$ for cold stream $j$ ). In this way, the energy balances for each hot and cold stream around each heat exchanger can be combined into overall heat balances per stage that are linear. Assignment constraints are used to link the temperatures at the superstructure with the inlet temperatures. Energy balances for the final utility units define the utility loads for each period. To ensure feasibility of temperatures, it is specified monotonic temperature decrease along the stages. Upper bound constraints are needed to relate the heat loads $q$ with the binary variables $z$. The parameter $\Omega$ is an upper bound for the corresponding heat load. If the heat load is not equal to zero the corresponding binary variable is set to one, otherwise the binary variable can be either 0 or 1 , but the objective function forces the variable to be zero in order to minimize the number of units. 
In addition, big-M constraints are needed to ensure that the temperature approaches only hold if the heat exchanger exists. The parameter $\Gamma$ is an upper bound for the temperature difference. If the binary variable is equal to zero the equations are ensured to be feasible. On the other hand, if the binary variable is equal to one, the temperature differences are forced to act as an equality constraint in order to minimize the areas in the objective function. To ensure minimum driving forces, the temperature differences are forced to be greater than $\Delta T_{\min }$. The logarithmic mean temperature difference is replaced by the Chen approximation (Chen, 1987) in order to avoid numerical difficulties. The required area for process-process heat exchangers can be calculated by inequality constraints. It is important to notice that due to the direction of the objective function, these inequality constraints are forced to be active for the worst case where the maximum area occurs. Finally, the objective function is the total annual cost (TAC) as a result of the average utility consumption and the investment cost.

\subsection{Flexibility Index Evaluation}

For a given design, the operation can be described by a set of equality constraints $I$ and inequality constraints $J$, representing the plant operation and design specifications:

$$
\begin{aligned}
& h_{i}(d, z, x, \theta)=0, \quad i \in I \\
& g_{j}(d, z, x, \theta) \leq 0, \quad j \in J
\end{aligned}
$$

where $d$ corresponds to the vector of design variables, $z$ the vector of control variables, $x$ the states variables and $\theta$ the vector of uncertain parameters. As has been shown by Halemane and Grossmann (1983), for a specific design, $d$, given this set of constraints, the design feasibility test problem can be formulated as the max-min-max problem:

$$
\chi(d)=\max _{\theta \in T} \min _{z} \max _{j \in J}\left\{\begin{array}{r}
g_{j}(d, z, x, \theta) \leq 0 \\
\text { s.t. } h_{i}(d, z, x, \theta)=0
\end{array}\right\}
$$

where the function $\chi(d)$ represents a feasibility measure for design $d$. If $\chi(d) \leq 0$, design $d$ is feasible for all $\theta \in T$, whereas if $\chi(d)>0$, the design cannot operate for at least some values of $\theta \in T$. The above max-min-max problem defines a nondifferentiable global optimization problem, which however can be reformulated as the following two-level optimization problem: 


$$
\begin{cases}\chi(d)= & \max _{\theta \in T} \psi(d, \theta) \\ \text { s.t. } & \psi(d, \theta) \leq 0 \\ & \psi(d, \theta)=\min _{z} u \\ & \text { s.t. } h_{i}(d, z, x, \theta)=0 \\ & g_{j}(d, z, x, \theta) \leq u\end{cases}
$$

where the function $\psi(d, \theta)$, denoted as the feasibility function, defines the boundary of the feasible region in the space of the uncertain parameters $\theta$. This function projects the space $d, z, x, \theta$ into the space $d, \theta$. Its value is negative inside, zero at the boundary and strictly positive outside the feasible region.

Plant feasibility can be quantified by determining the flexibility index of the design. Following the definition of the flexibility index proposed by Swaney and Grossmann (1985), this metric expresses the largest scaled deviation $\delta$ of any expected deviation $\Delta \theta^{+}, \Delta \theta^{-}$in positive and negative direction that the design can handle for feasible operation. The mathematical formulation for the evaluation of the design's flexibility is the following:

$$
\left\{\begin{array}{c}
F=\max \delta \\
\text { s.t. } \quad \chi(d)=\max _{\theta \in T} \min _{z} \max _{\substack{i \in I \\
j \in J}}\left\{\begin{array}{c}
g_{j}(d, z, x, \theta) \leq 0 \\
\text { s.t. } h_{i}(d, z, x, \theta)=0
\end{array}\right\} \leq 0 \\
T(\delta \geq 0)=\left\{\theta \mid \theta^{N}-\delta \Delta \theta^{-} \leq \theta \leq \theta^{N}+\delta \Delta \theta^{+}\right\}
\end{array}\right.
$$

The design flexibility index problem in (5) can be reformulated to represent the determination of the largest scaled hyperrectangle that can be inscribed within the feasible region. Following this idea, the mathematical formulation of the flexibility index problem has the following form:

$$
\left\{\begin{array}{l}
\begin{array}{l}
F=\min \delta \\
\text { s.t. } \quad
\end{array} \quad \begin{array}{l}
\psi(d, \theta)=0 \\
\psi(d, \theta)=\min _{x, z} u
\end{array} \\
\text { s.t. } \quad h_{i}(d, z, x, \theta)=0 \\
\quad g_{j}(d, z, x, \theta) \leq u \\
T(\delta \geq 0)=\left\{\theta \mid \theta^{N}-\delta \Delta \theta^{-} \leq \theta \leq \theta^{N}+\delta \Delta \theta^{+}\right\}
\end{array}\right.
$$

The problems (4) and (6) are bi-level optimization problems. An outer problem defining the feasibility test/flexibility index, and an inner problem to for the feasibility function evaluation. For the case where the constraints $h_{i}$ and $g_{j}$ are convex, Swaney and Grossmann (1985) proved that the point $\theta^{c}$ that defines the solution of (4) lies at 
one of the vertices of the parameter set $T$. Based on this assumption, the outer problem is evaluated for each possible solution of the inner problem, i.e. the inner problem evaluated at each vertex. The main issue is that the number of vertices may be large.

To circumvent this limitation, and also solve for nonconvex problems, an active set strategy was proposed by Grossmann and Floudas (1987) based on the following ideas: (i) the inner optimization problem in (4) and (6) is replaced by the Karush-Kuhn-Tucker optimality conditions (KKT); (ii) it is assumed that under certain conditions (assumed to be valid in this work) the number of active constraints is equal to the number of control variables plus one $\left(n_{z}+1\right)$; and (iii) the discrete nature of the selection of the active constraints is performed by introducing a set of binary variables $y_{j}$ to represent if the constraint $g_{j}$ is active. Based on these ideas, the feasibility test and the flexibility test problem can be reformulated in the following way,

Feasibility Test:

$$
\left(P 2^{\prime}\right)\left\{\begin{array}{l}
\chi(d)=\max u \\
\text { s.t. } h_{i}(d, z, x, \theta)=0 \\
g_{j}(d, z, x, \theta)+s_{j}-u=0 \\
\sum_{j \in J} \lambda_{j}=1 \\
\sum_{j \in J} \lambda_{j} \frac{\partial g_{j}}{\partial z}+\sum_{i \in I} \mu_{i} \frac{\partial h_{i}}{\partial z}=0 \\
\sum_{j \in J} \lambda_{j} \frac{\partial g_{j}}{\partial x}+\sum_{i \in I} \mu_{i} \frac{\partial h_{i}}{\partial x}=0 \\
\lambda_{j}-y_{j} \leq 0, j \in J \\
s_{j}-U\left(1-y_{j}\right) \leq 0, j \in J \\
\sum_{j \in J} y_{j}=n_{z}+1 \\
\theta^{L} \leq \theta \leq \theta^{U} \\
\delta \geq 0 \\
y_{j}=\{0,1\}, \quad \lambda_{j}, s_{j} \geq 0, j \in J
\end{array}\right.
$$

\section{Flexibility Test:}

$F=\min \delta$

s.t. $h_{i}(d, z, x, \theta)=0$

$g_{j}(d, z, x, \theta)+s_{\mathrm{j}}-u=0$

$u=0$

$\sum_{j \in J} \lambda_{j}=1$

$\sum_{j \in J} \lambda_{j} \frac{\partial g_{j}}{\partial z}+\sum_{i \in I} \mu_{i} \frac{\partial h_{i}}{\partial z}=0$

$\sum_{j \in J} \lambda_{j} \frac{\partial g_{j}}{\partial x}+\sum_{i \in I} \mu_{i} \frac{\partial h_{i}}{\partial x}=0$

$\lambda_{j}-y_{j} \leq 0, j \in J$

$s_{j}-U\left(1-y_{j}\right) \leq 0, j \in J$

$\sum_{j \in J} y_{j}=n_{z}+1$

$\theta^{N}-\delta \Delta \theta^{-} \leq \theta \leq \theta^{N}+\delta \Delta \theta^{+}$

$\delta \geq 0$

$y_{j}=\{0,1\}, \quad \lambda_{j}, s_{j} \geq 0, j \in J$

where $s_{\mathrm{j}}$ are slack variables of constraints $g_{j}, \lambda_{j}, \mu_{i}$, are the Lagrangean multipliers for inequality and equality constraints, respectively, and $U$ represents an upper bound to the slack variables $s_{j}$. These problems correspond to mixed integer optimization problems 
either linear or nonlinear MI(N)LP depending on the nature of the constraints (1) and (2).

In order to carry out the flexibility analysis for HENs designed by the model (P1), all relevant equality and inequality for the HEN operation considered here is given by:

and

$$
h(d, z, x, \theta)=\left\{\begin{array}{c}
\sum_{\forall j \in C P} q_{i j k}-\left(t_{i k}-t_{i, k+1}\right) F_{i} \\
\sum_{\forall i \in H P} q_{i j k}-\left(t_{j k}-t_{j k+1}\right) F_{j} \\
q_{c u i}-\left(t_{i, N_{T}+1}-T_{i}^{o u t}\right) F_{i} \\
q_{h u j}-\left(T_{j}^{\text {out }}-t_{j 1}\right) F_{j} \\
T_{i}^{\text {in }}-t_{i 1} \\
T_{j}^{\text {in }}-t_{j, N_{T}+1}
\end{array}\right\}=0
$$

$$
g(d, z, x, \theta)=\left\{\begin{array}{c}
t_{i, k+1}-t_{i k} \\
t_{j, k+1}-t_{j k} \\
T_{i}^{\text {out }}-t_{i, N_{T}+1} \\
t_{j 1}-T_{j}^{\text {out }} \\
\Delta T_{\text {min }}+t_{j k}-t_{i k} \\
\Delta T_{\text {min }}+t_{j, N_{T}+1}-t_{i, N_{T}+1} \\
\Delta T_{\text {min }}+T_{c u}^{\text {out }}-t_{i, N_{T}+1} \\
\Delta T_{\text {min }}+t_{j 1}-T_{h u}^{\text {out }}
\end{array}\right\} \leq 0
$$

Substituting the equations (7) and (8) in the formulation described (either vertex search or active set strategy) it is possible to solve the feasibility tested and flexibility index problem. It should be noted that these equations are based on the constraints of SYNHEAT model, and the overall heat balances are not included because they can be obtained by combining other independent equalities. It ensures the full rank of the partial derivatives of the constraints with respect to the control variables $z$, which is a premise of the active set strategy. The control variables are chosen as the degrees of freedom during operation, determined by the number of equations minus the number of unknown variables. It is important to be aware that during the flexibility analysis all degrees of freedom (potential manipulations for control) are used to achieve feasible operation. 


\subsection{Control Structure Selection}

For a given flexible design and a given number of controlled variables, assuming a decentralized control structure is implemented for operation, each controlled variable is paired with one manipulated variable selected from a given set of potential choices. Each pair defines a control loop and the whole set of pairs form the Control Structure (CS). A more recent review for CS selection can be found in Wal and Jager (2001). The controllability and the effective flexibility depend on the CS. A large amount of literature on CS selection (also referred as IO selection) is devoted to quantitative measures for controllability. In this work we mainly considered two major metrics for controllability: (i) the Relative Gain Array (RGA) as a measure of the interaction between the control loops; and (ii) the disturbance sensitivity (DS) as a measure of the ability of a specific control structure to reject disturbances. A CS is desirable with low interaction and with small disturbance sensitivity.

Kookos and Perkins (2001) proposed an MILP formulation for the control structure selection based on RGA and DS. In order to apply the proposed approach it is necessary to obtain the transfer matrix model of the process. Consider the system described by the following transfer function matrices:

$$
y(s)=G(s) u(s)+G_{d}(s) d(s)
$$

where $\mathrm{y}(\mathrm{s})$ is the Laplace transform of the $n y$ vector of controlled variables $y(t), u(s)$ is the Laplace transform of the $n u$ vector of potential manipulated variables $u(t)$, and $d(s)$ is the Laplace transform of the $n d$ vector of disturbances $d(t)$.

The matrix $G(s)$ relates the set of potential manipulated variables $j \in M V=\{1, \ldots, n u\}$ with the controlled variables over the set $i \in C V=\{1, \ldots, n y\}$ where $g_{i j}$ points out the stationary gain element of $G(0)$. We can define the matrix $X$ :

$$
X_{i j}=\left[\begin{array}{cc}
1, & \text { if manipulated } j \text { is used to control controlled variable } \dot{\eta} \\
0, & \text { otherwise }
\end{array}\right]
$$

\subsubsection{Minimization of the Interaction}


The Relative Gain Array (RGA) was introduced by Bristol (1966) and is commonly used as a measure of interaction, an important aspect of integrated process. For any feasible selection of $n y$ manipulated inputs $(n y \leq n u)$, the square matrix of dimension $n y \times n y$, the RGA is defined by:

$$
R G A=G(0) \times\left[G^{-1}(0)\right]^{T}
$$

For the calculation of the RGA, linear equations were proposed. The square matrix of dimension $n y \times n y(\widehat{G})$ that consists of the $n y$ columns of the $G$ matrix that correspond to these inputs must be invertible. The matrix $\tilde{G} n y \times n u$ includes the columns of the transpose inverse of $\hat{G}$ plus $n u-n y$ zero columns. The elements of this non square matrix $\tilde{g}_{i j}$ are defined as follows:

$$
\sum_{i=1}^{n u} g_{i j} \tilde{g}_{\ell j}-\delta_{i \ell}=0, \quad \forall i, \ell
$$

where $\delta_{i \ell}$ is the Kronecker delta, and $\ell$ is an index for a set over the controlled variables. To enforce the fact that the columns for wich the sum $\sum_{i=1}^{n y} X_{i j}$ is zero have to have zero elements, Big-M constraints are added to the model:

$$
-\Omega \sum_{i=1}^{n y} X_{i j} \leq \tilde{g}_{\ell j} \leq \Omega \sum_{i=1}^{n y} X_{i j}, \quad \forall i, j
$$

where $\Omega$ is a sufficiently large number. The element $R G A \lambda_{i j}$ can be computed as

$$
\lambda_{i j}-g_{i j} \tilde{g}_{i j}=0, \quad \forall i, j
$$

In addition, the following constraints are included to denote the fact that one and only one manipulated variable has to be assigned to each controlled output:

$$
\begin{aligned}
& \sum_{i=1}^{n y} X_{i j}-1 \leq 0, \quad \forall j \\
& \sum_{j=1}^{n u} X_{i j}-1=0, \quad \forall i
\end{aligned}
$$

Input and output variables in a control loop should be paired so that the diagonal elements of RGA are close to one as possible. Thus, the promising sets of manipulated 
variables and pairings is based on the minimization of the RGA Number (Skogestad and Postlethwaite, 1996):

$$
R G A \text { Number }=\|R G A-I\|_{\text {sum }}=\sum_{i=1}^{n y} \sum_{j=1}^{n u}\left|\lambda_{i j}-X_{i j}\right|
$$

Defining the auxiliary variables $\eta_{i j}=\lambda_{i j}-X_{i j}$ and $\mu_{i j}$ as the absolute value of $\eta_{i j}$ $\left(-\mu_{i j} \leq \eta_{i j} \leq \mu_{i j}\right)$. The MILP formulation used to solve the control structure selection that minimizes the overall interactions (Kookos and Perkins, 2001) is given by:

$$
\begin{aligned}
\min & \Lambda=\sum_{i=1}^{n y} \sum_{j=1}^{n u} \mu_{i j} \\
& \sum_{i=1}^{n y} X_{i j}-1 \leq 0 \\
& \sum_{j=1}^{n u} X_{i j}-1=0 \\
& \sum_{i=1}^{n u} g_{i j} \tilde{g}_{\ell j}-\delta_{i \ell}=0, \quad \forall i, \ell \\
n y & \left\{\begin{array}{c}
n \sum_{i=1}^{n} X_{i j} \leq \tilde{g}_{\ell j} \leq \Omega \sum_{i=1}^{n y} X_{i j} \\
\lambda_{i j}-g_{i j} \tilde{g}_{i j}=0 \\
\eta_{i j}=\lambda_{i j}-X_{i j} \\
-\mu_{i j} \leq \eta_{i j} \leq \mu_{i j} \\
X_{i j}=\{0,1\}, \quad \mu_{i j} \geq 0 \quad \forall i, j
\end{array}\right\} \forall i, j
\end{aligned}
$$

\subsubsection{Minimization of the Sensitivity to Disturbances}

A further objective that can be posed in the control structure selection problem is the DS. A measure of the ability of a specific control structure to reject disturbances is the disturbance sensitivity defined as follows:

$$
D S=\left\|\hat{G}^{-1}(0) G_{d}(0)\right\|_{\infty}
$$

Defining the matrix

$$
S=\widehat{G}^{-1}(0) G_{d}(0)
$$

and also the $n u \times n d$ matrix $\Sigma$ defined by the equation: 


$$
\sum_{i=1}^{n y} \tilde{g}_{i j} g_{d, i m}-\sigma_{j m}=0, \quad \forall j, m
$$

where $m \in D V=\{1, \ldots, n d\}$. The matrix $\Sigma$ consist of the $n y$ rows of the $S$ matrix that correspond to the inputs selected in the structure, as well as $n u-n y$ additional zero rows that correspond to the inputs not selected in the structure. Because of this property, Kookos and Perkins (2001) showed that the equation $\|S\|_{\infty}=\|\Sigma\|_{\infty}$ holds true. The MILP formulation for finding the set of manipulated inputs with minimum disturbance sensitivity is as follows:

$$
\begin{aligned}
& \min \varphi \\
& \sum_{i=1}^{n y} X_{i j}-1 \leq 0 \\
& \sum_{j=1}^{n u} X_{i j}-1=0 \\
& \sum_{i=1}^{n u} g_{i j} \tilde{g}_{\ell j}-\delta_{i \ell}=0, \quad \forall i, \ell \\
& -\Omega \sum_{i=1}^{n y} X_{i j} \leq \tilde{g}_{\ell j} \leq \Omega \sum_{i=1}^{n y} X_{i j} j \\
& \sum_{i=1}^{n y} \tilde{g}_{i j} g_{d, i m}-\sigma_{j m}=0, \quad \forall j, m \\
& \left\{\begin{array}{c}
n d \\
-\epsilon_{j} \leq \sum_{m=1}^{n d} \sigma_{j m} \leq \epsilon_{j} \\
\epsilon_{j}-\varphi \leq 0
\end{array}\right\} \\
& X_{i j}=\{0,1\}, \quad \mu_{i j} \geq 0, \quad \epsilon_{j} \geq 0 \forall i, j
\end{aligned}
$$

The formulation (S2) does not provide an answer to the input-output pairing problem. It is due the fact that the disturbance sensitivity measure does not depend on the pairing, but only on the sets of controlled and manipulated variables selected. To simultaneously consider the interactions and the disturbance sensitivity, the formulation (S3) combining (S1) and (S2) objective function can be used $(\rho \Lambda+(1-\rho) \varphi)$. The parameter $\rho$ is the weighting coefficient $(0<\rho \leq 1)$ used to assign different contributions for the metrics of interaction and disturbance sensitivity to the objective function. 


\subsubsection{Additional Constraints}

In the previous subsection an MILP formulation for promising control structures was presented. These models were proposed for a general process. In order to apply them to HENs some qualitative knowledge was incorporated to the model. It is assumed that each heat exchanger has a bypass on either cold or hot side. Both are considered as a potential manipulation for the control structure. However, since we have only one degree of freedom per exchanger they cannot be selected simultaneously, which is enforced by the following constraint:

$$
\sum_{i=1}^{n y} X_{i j}+\sum_{i=1}^{n y} X_{i j}{ }^{\prime} \leq 1, \quad\left\{j, j^{\prime}\right\} \in H E
$$

where $H E$ is the set of manipulations $j$ and $j$ ' associated with the same heat exchanger. In addition, it is desirable to identify whenever the heat exchanger is bypassed or not. One binary variable is defined $\left(y e_{k x}\right)$ that is one if the heat exchanger $k x$ is bypassed, zero otherwise. It is assumed the heat exchanger $k x$ is associated with a pair of potential manipulations $H E_{k x}=\left\{j, j^{\prime}\right\}$. We can derive the constraints (22) and (23). The former states that if one side of the heat exchanger is bypassed, then the exchanger is bypassed, i.e. $y e_{k x}=1$, and the later states that if none side is bypassed, then $y e_{k x}$ is forced to be zero.

$$
y e_{k x} \geq \sum_{i=1}^{n y} X_{i j}, j \in H E_{k x}
$$

and

$$
y e_{k x} \leq \sum_{i=1}^{n y} X_{i j}+\sum_{i=1}^{n y} X_{i j^{\prime}},\left\{j, j^{\prime}\right\} \in H E_{k x}
$$

\subsection{Effective Flexibility Index}

In subsection 3.2, it was assumed that all degrees of freedom can be adjusted during the operation. However, it is important to be aware that the actual flexibility may be limited by the control strategy. Even if the HEN is sufficiently flexible, there is no guarantee that the control strategy will adjust the manipulations such that full flexibility is 
attainable. Therefore, the term effective flexibility here is denoted as the ability of a controlled HEN to maintain feasible steady-state operation for the given uncertainty range. In this way, the effective flexibility is not only a property of the network structure by itself as the flexibility, but it also depends on the control structure. It is also important to note that the flexibility index is an upper bound for the effective flexibility, and they are equivalent if the number of control variables $n z$ is equal to zero. In fact, it means that there is no extra degree of freedom since all of them were consumed by the control system.

In order to compute the effective flexibility some minor modifications in the original model (P2) are necessary to include the information about the control structure. Each heat exchanger contributes with two potential manipulations. For the heat exchangers in which the bypass is not selected for the control structure, the bypass fraction must stay fixed at its optimal value $\left(u^{*}\right)$ defined at the design stage. It would be possible to enforce one assignment constraint $\left(u=u^{*}\right)$. However, the bypass is not explicitly modeled in problem (P1). To overcome this issue an alternative procedure was used where we recognize that for the subset of heat exchangers where we cannot adjust its bypass fraction, the heat load and temperatures of this heat exchanger will not vary freely but approximately according to the expression $(q=U A L M T D)$ for fixed area defined in the design stage. This expression was approximated by the equation (24), for which the logarithmic mean temperature was replaced by the arithmetic mean in order to keep this constraint linear.

$$
q_{i j k}=\frac{1}{2} U A_{i j k}\left(d t_{i j k}+d t_{i j k+1}\right)
$$

If the heat exchanger is not bypassed, the above constraint must be added as an extra operation al constraint to the Flexibility Index problem. In order to link the constraints of the Control Structure Selection problem (S3) with the constraints of the Flexibility problem (P2) we have added the following Big-M constraints:

$$
\frac{1}{2} U A_{i j k}\left(d t_{i j k}^{p}+d t_{i j k}^{p}\right)-\Omega y e_{k x} \leq q_{i j k}^{p} \leq \frac{1}{2} U A_{i j k}\left(d t_{i j k}^{p}+d t_{i j k}^{p}\right)+\Omega y e_{k x}
$$


where $\Omega$ is an upper bound for the heat load. If the heat exchanger is not bypassed $\left(y e_{k x}=0\right)$ the constraint (25) reduces to (24), otherwise if $y e_{k x}=1$ the constraint (25) becomes redundant.

The operating stage can be solved sequentially where we first find the Control Structure solving model (S3) with minimum controllability metric, and then we solve the model (P2) for the effective flexibility for the CS adding constraints (24) for each heat exchanger that is not selected by Control Structure. While this procedure is easy to implement, however, it may exclude effectively flexible control structures with less favorable controllability. Alternatively, the models (S3) and (P2) may be solved simultaneously. The set of constraints is linked by the constraints (22), (23) and (25), and the resulting multiobjective problem (P4) is formulated using the $\varepsilon$-constraint method to define the effective flexibility as follows,

$$
(P 4)\left\{\begin{aligned}
E F= & \min \delta \\
\text { s.t. } & (P 2) \text { constraints } \\
& (S 3) \text { constraints } \\
& (21),(22),(23),(25) \\
& \rho \Lambda+(1-\rho) \varphi \leq \varepsilon
\end{aligned}\right.
$$

where $\varepsilon$ is an upper bound for the controllability metric. The minimum feasible value for $\varepsilon$ corresponds to the effective flexibility index for the control structure selection with minimum controllability metric. On the other hand, a sufficient large value of $\varepsilon$ corresponds the effective flexibility index for a feasible control structure. The set of solutions between these points defines the Pareto curve. Since the priority is to find feasible operation for a given control structure, i.e. $E F \geq 1$, large values of $\varepsilon$ may be used. We can solve this problem for different instances decreasing the value of $\varepsilon$ until we have an effective flexibility index $E F$ greater than one. If we solve the problem (P4) for a sufficient large $\varepsilon$ and the flexibility is not greater than one, there is no effective flexible control structure. 


\section{Outline of the solution strategy}

The outline of the solution strategy is sketched in Figure 6. The procedure starts with the design for selected conditions (e.g. nominal conditions), for which the multiperiod problem (P1) is reduced to the SYNHEAT model. The problem (P2) is solved in order to evaluate the Flexibility index for the given design. If the design is not flexible, i.e. the flexibility index is not greater than one, the critical point that defines the solution of (P2) is added to the nominal conditions and the problem (P1) is solved again, with nominal conditions and the critical point. Otherwise the static model is generated for the design and the MILP problem (S3) is solved for the control structure selection.

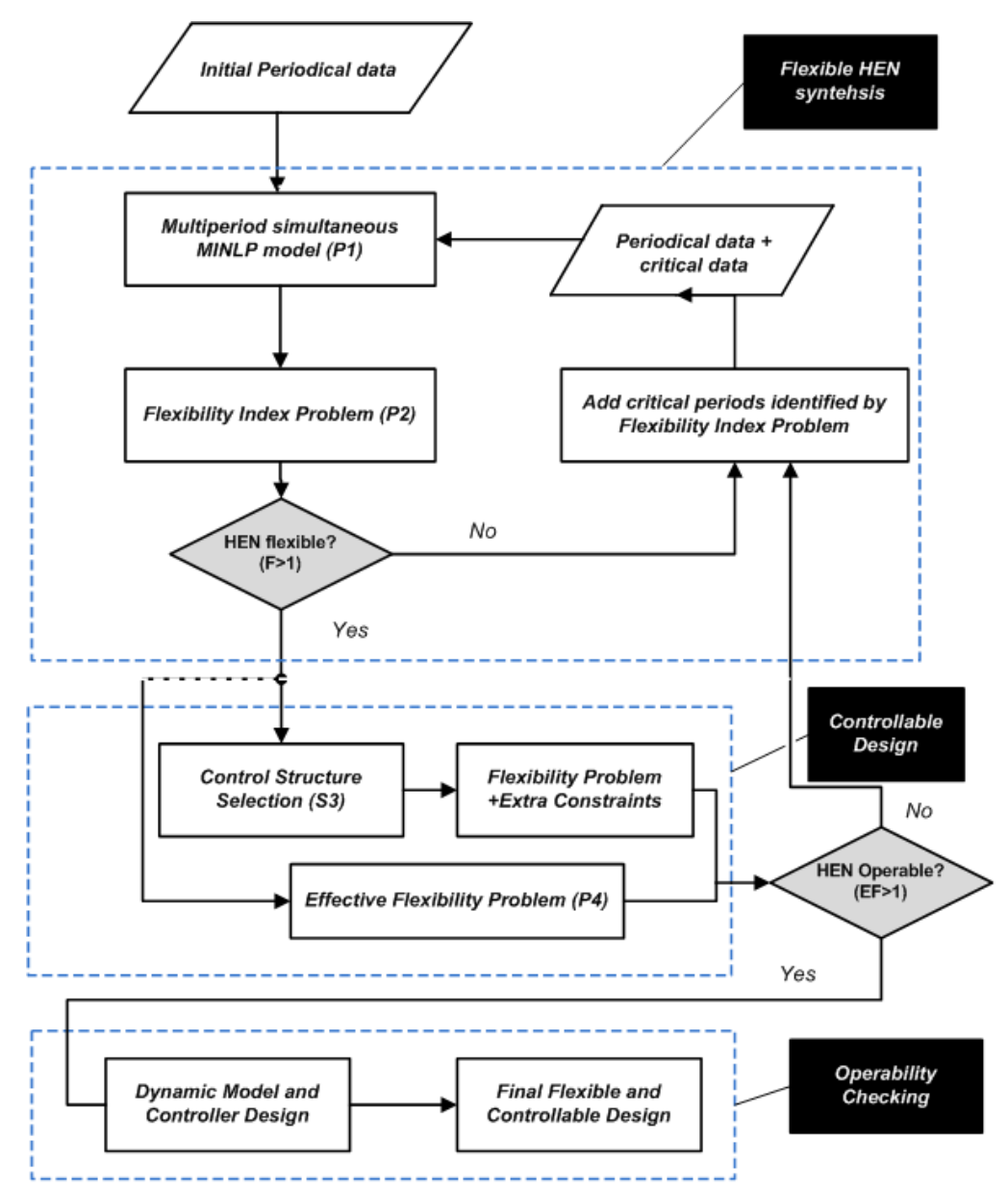

Figure 6. Outline of the solution strategy.

This problem (S3) may have multiple solutions. In order to obtain all alternative solutions integer cuts are added cumulatively to prevent the same solution to repeat again until there is a change in the objective function. For each control structure, we can 
evaluate the effective flexibility index to find the structures that could be implemented to reject the disturbance level. Alternatively, we can solve problem (P4) to find the effective flexibility and the control structure simultaneously. Once we have found the optimal design with minimum TAC, and able to remain feasible under uncertain conditions for a given control structure, it is possible to generate the dynamic model and design low order controllers, and then check dynamic performance of the closed loop.

\section{Numerical Examples}

Three examples are given in this section to illustrate the solution strategy for synthesizing flexible and controllable heat exchanger networks. The models were formulated using GAMS and solved on an Intel Core 2.67 GHz machine with 2.96 GB memory. GAMS/CONOPT 3.0 and BARON were used to solve the NLP problems, GAMS/CPLEX 10.0 was used for the MILP problems, and GAMS/DICOPT was employed for solving the MINLP problems. For the multiperiod optimization problem local solvers were used. On the other hand, for the Flexibility Index the problem was solved to optimality using BARON. The generation of the linearized steady-state and dynamic models, as well the closed loop simulations were performed in Matlab.

\subsection{Example 1}

The first numerical example involves two hot streams and two cold streams. The nominal data for the problem is listed in Table 1. Expected variations in the inlet temperatures of $\delta_{T}=10 K$ are assumed, a $\Delta \mathrm{T}_{\min }$ of $10 \mathrm{~K}$ and the minimum number of stages was set to two.

Table 1. Problem data for example 1.

\begin{tabular}{ccccc}
\hline Stream & $\begin{array}{c}T_{\text {in }} \\
(\mathrm{K})\end{array}$ & $\begin{array}{c}T_{\text {out }} \\
(\mathrm{K})\end{array}$ & $\begin{array}{c}F \\
\left(\mathrm{~kW} \cdot \mathrm{K}^{-1}\right)\end{array}$ & $\begin{array}{c}h \\
\left(\mathrm{~kW} \mathrm{~m}^{2} \mathrm{~K}^{-1}\right)\end{array}$ \\
\hline H1 & $583 \pm 10$ & 323 & 1.4 & 0.16 \\
$\mathrm{H} 2$ & $723 \pm 10$ & 553 & 2.0 & 0.16 \\
$\mathrm{C} 1$ & $313 \pm 10$ & 393 & 3.0 & 0.16 \\
$\mathrm{C} 2$ & $388 \pm 10$ & 553 & 2.0 & 0.16 \\
$\mathrm{CU}$ & 303 & 323 & & 016 \\
$\mathrm{HU}$ & 573 & 573 & 0.16 \\
\hline Cost of Heat Exchangers $\left(\$ \mathrm{y}^{-1}\right)=5500+4333\left[\text { Area }\left(\mathrm{m}^{2}\right)\right]^{0.6}$ & \\
Cost of Cooling Utility $=60.576\left(\$ \mathrm{~kW}^{-1} \mathrm{y}^{-1}\right)$ & & \\
Cost of Heating Utility $=171.428\left(\$ \mathrm{~kW}^{-1}-1 \mathrm{y}-1\right)$ &
\end{tabular}


Initially, the example was solved for the nominal data using the model (P1) resulting in the design depicted in Figure 7 with a Total Annual Cost (TAC) of \$ 92,210 per year. The three process to process heat exchangers were identified with a tag number (hot, cold, stage). It can be seen that the maximum heat integration is achieved since there is only one cold utility exchanger and no hot utility consumption.

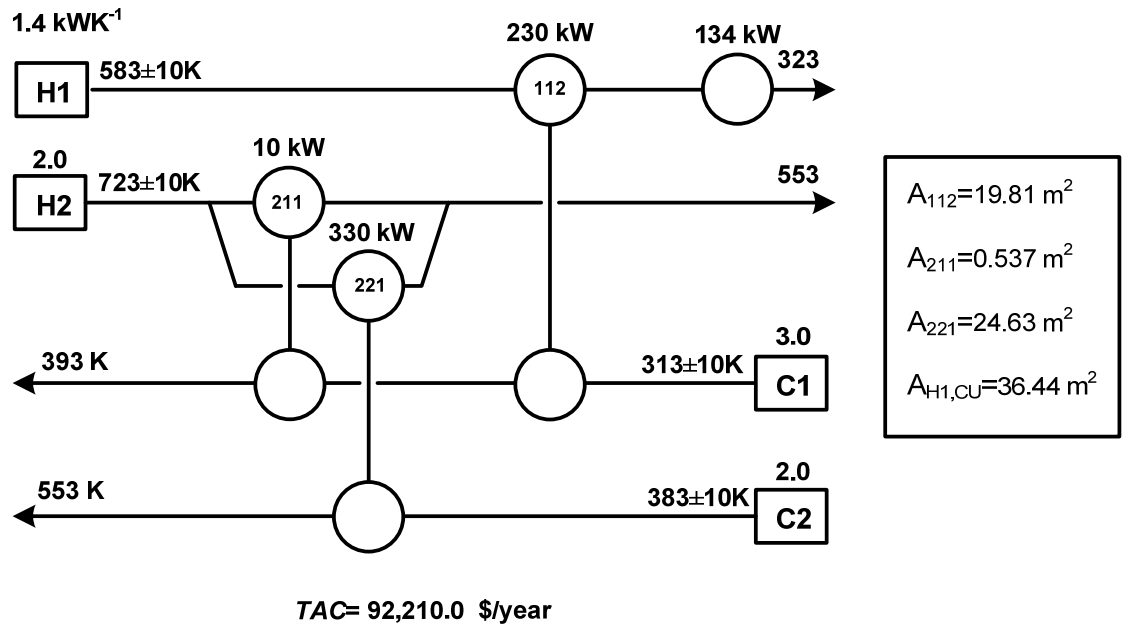

Figure 7. Nominal Design for Example 1.

For this example the steady-state model was developed, i.e. the gain matrices for disturbances (inlet temperatures variations $\delta T^{i n}$ and heat capacity flowrates $\delta F$ ) and for each potential manipulation (bypass either on hot $\delta u^{H}$ or cold side $\delta u^{C}$ of each heat exchanger) to the controlled outlet temperatures $\delta T^{\text {out }}$. For the given heat load distribution, a subroutine written in Matlab generates the following model:

$$
\begin{aligned}
{\left[\begin{array}{l}
\delta T_{H 1}^{\text {out }} \\
\delta T_{H 2}^{\text {out }} \\
\delta T_{C 1}^{\text {out }} \\
\delta T_{C 2}^{\text {out }}
\end{array}\right]=} & {\left[\begin{array}{cccccc}
49.98 & 0 & 0 & 23.32 & 0 & 0 \\
-0.35 & 1.27 & 41.86 & -0.16 & 0.025 & 40.63 \\
-23.09 & -0.85 & 0 & -10.78 & -0.017 & 0 \\
0 & 0 & -41.86 & 0 & 0 & -40.63
\end{array}\right]\left[\begin{array}{l}
\delta u_{112}^{H} \\
\delta u_{211}^{H} \\
\delta u_{221}^{H} \\
\delta u_{112}^{C} \\
\delta u_{211}^{C} \\
\delta u_{221}^{C}
\end{array}\right] } \\
& +\left[\begin{array}{ccccc}
0.391 & 0 & 0.608 & 0 \\
0.004 & 0.49 & 0.011 & 0.492 \\
0.281 & 0.01 & 0.709 & 0 \\
0 & 0.49 & 0 & 0.507
\end{array}\right]\left[\begin{array}{l}
\delta T_{H 1}^{\text {in }} \\
\delta T_{H 2}^{\text {in }} \\
\delta T_{C 1}^{\text {in }} \\
\delta T_{C 2}^{\text {in }}
\end{array}\right] \\
+ & {\left[\begin{array}{cccc}
81.64 & 0 & -7.775 & 0 \\
0.249 & 63.42 & -0.337 & -20.31 \\
16.493 & 0.425 & -22.813 & 0 \\
0 & 20.932 & 0 & -62.18
\end{array}\right]\left[\begin{array}{l}
\delta F_{H 1} \\
\delta F_{H 2} \\
\delta F_{C 1} \\
\delta F_{C 2}
\end{array}\right] }
\end{aligned}
$$


With the linearized model (Eq. 26) it is possible to estimate the impact of the disturbances on the controlled variables, and also select control structures with the best controllability properties based only on static knowledge. It can be noted that disturbances in the hot stream $\mathrm{H} 2$ and cold stream $\mathrm{C} 2$ will not affect the outlet temperatures of hot stream H1, since there is no downstream path (an unbroken connection) evidenced by the zero gain. This is in accordance with the structure depicted in Figure 8. From the control structure point of view, only a bypass on heat exchanger 221 for hot stream $H 2$ can be selected to control the cold stream $C 2$, since there are structural singularities from the other potential manipulations.

With the model (26), the problem (S1) was solved for finding the control structure with minimum overall interaction. Multiple solutions were obtained by using integer cuts. A total of eight structures were found, all of them with RGA number of zero. Two possible control structures are sketched in Figure 8.

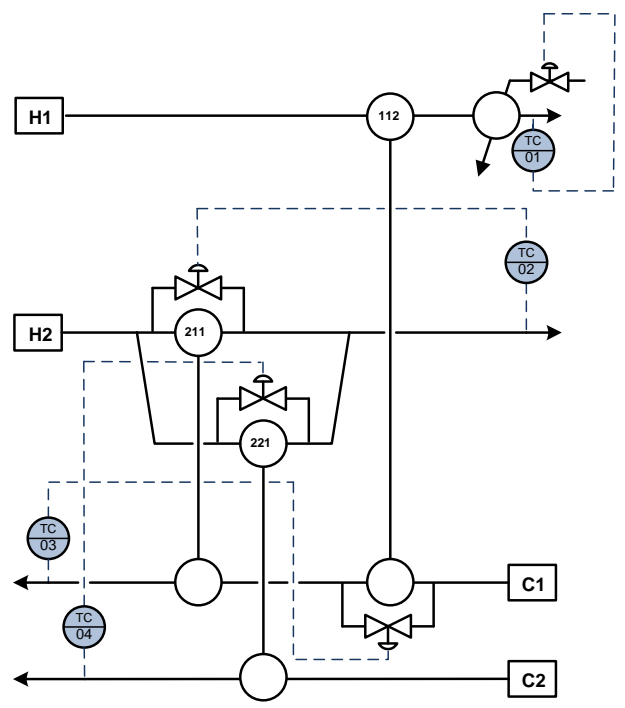

(a)

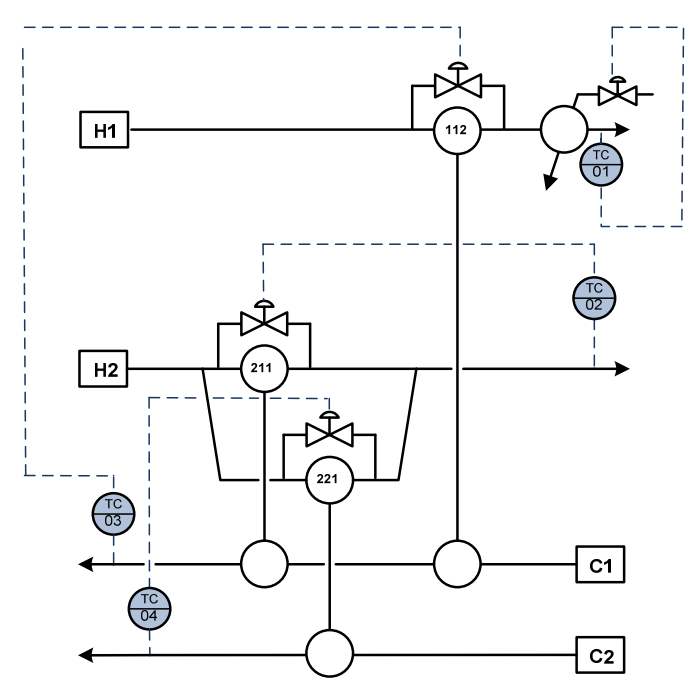

(b)

Figure 8. Two possible Control Structures for Nominal Design.

For the nominal design, there are no control variables $(n z=0)$. Therefore, all heat exchanger units must be used in order to control the network. In order words, all possible independent manipulations are consumed by the control structure. For this particular case, there is no distinction between the Flexibility Index and the Effective Flexibility Index, and a value of 0.25 was found pointing out that the design is not 
flexible enough in order to handle the specified disturbance level. In fact, one could say that the nominal design can remain feasible only for variations of $2.5 \mathrm{~K}$ in the inlet temperatures, instead of the expected $10 \mathrm{~K}$. The critical point identified at the solution of the problem (P2) is added to the nominal condition and the multiperiod optimization problem (P1) is performed again considering these two periods. The general results of the two-stage strategy are listed in Table 2 and the periods considered in the design are given in Table 3 . The TAC of the new configuration is $\$ 130,474$ per year and a Flexibility Index of 1.429. It is important to mention that an optimal flexibility degree may be achieved solving the two-stage strategy for different flexibility target $(\delta)$. However, as will be shown it is desirable some overestimation of the FI due to limitations imposed by the control structure. The configuration is depicted in Figure 9. In order to accomplish flexibility the new configuration has four process to process heat exchangers, which includes one extra control variable $(n z=1)$.

Table 2. General Results for TSS applied to Example 1.

\begin{tabular}{ccccc}
\hline Iter. & $\begin{array}{c}\text { Operating } \\
\text { Cost }(\$ / y r)\end{array}$ & $\begin{array}{c}\text { Investment } \\
\text { Cost }(\$ / y r)\end{array}$ & $\begin{array}{c}\text { Total Annual } \\
\text { Cost }(\$ / y r)\end{array}$ & $\begin{array}{c}\text { Flexibility } \\
\text { Index }\end{array}$ \\
\hline 1 & 8117 & 84093 & 92210 & 0.250 \\
2 & 5573 & 124901 & 130474 & 1.429 \\
\hline
\end{tabular}

Table 3. Uncertain Parameters for the Points considered.

\begin{tabular}{ccccc}
\hline Iter. & $T_{\text {in }}^{H 1}(K)$ & $T_{\text {in }}^{H 2}(K)$ & $T_{\text {in }}^{C 1}(K)$ & $T_{\text {in }}^{C 2}(K)$ \\
\hline 1 & 583 & 723 & 313 & 388 \\
2 & 573 & 713 & 303 & 378 \\
\hline
\end{tabular}

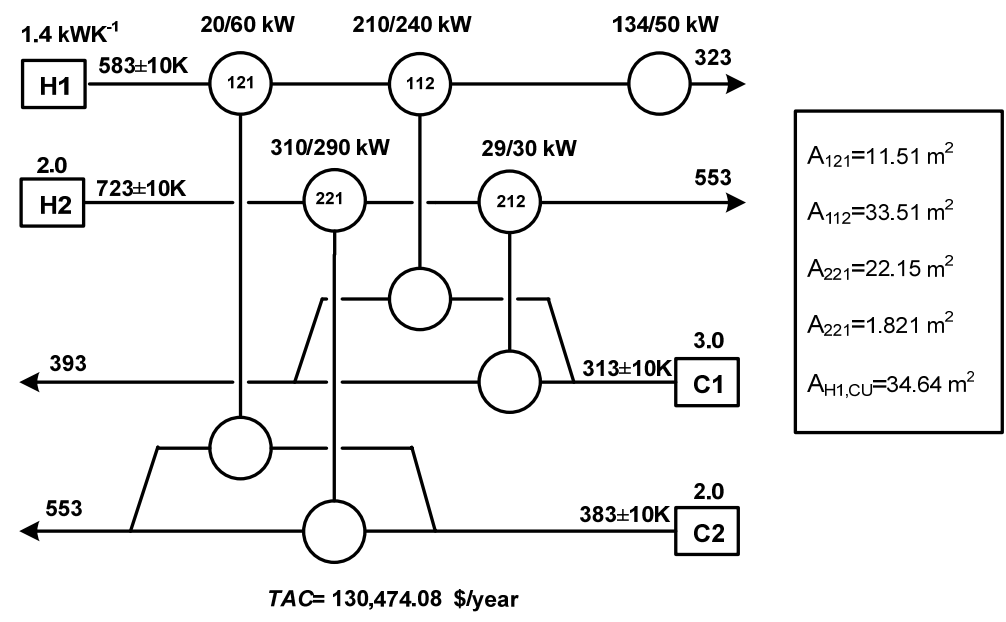

Figure 9. Flexible Configuration for Example 1. 
The steady-state model for the flexible configuration is as follows:

$$
\begin{gathered}
{\left[\begin{array}{l}
\delta T_{H 1}^{\text {out }} \\
\delta T_{H 2}^{\text {out }} \\
\delta T_{C 1}^{\text {out }} \\
\delta T_{C 2}^{\text {out }}
\end{array}\right]=\left[\begin{array}{cccccccc}
0.22 & 43.99 & 0 & 0 & 2.50 & 23.46 & 0 & 0 \\
0 & 0 & 33.75 & 0.44 & 0 & 0 & 35.93 & 2.35 \\
0.14 & -20.5308 & 1.401 & -0.29412 & 1.65 & -10.95 & 1.49 & -1.57 \\
-0.36 & 0 & -35.86 & 0 & -4.23 & 0 & -38.17 & 0
\end{array}\right]\left[\begin{array}{l}
\delta u_{121}^{H} \\
\delta u_{112}^{H} \\
\delta u_{221}^{H} \\
\delta u_{212}^{H} \\
\delta u_{121}^{C} \\
\delta u_{112}^{C} \\
\delta u_{221}^{C} \\
\delta u_{212}^{C}
\end{array}\right]} \\
+\left[\begin{array}{ccccc}
0.383 & 0 & 0.586 & 0.030 \\
0 & 0.506 & 0.058 & 0.435 \\
0.253 & 0.021 & 0.687 & 0.038 \\
0.051 & 0.463 & 0 & 0.486
\end{array}\right]\left[\begin{array}{l}
\delta T_{H 1}^{\text {in }} \\
\delta T_{H 2}^{\text {in }} \\
\delta T_{C 1}^{\text {in }} \\
\delta T_{C 2}^{\text {in }}
\end{array}\right]+\left[\begin{array}{ccccc}
79.782 & 0 & -7.821 & -1.249 \\
0 & 63.346 & -0.784 & -17.963 \\
17.356 & 2.483 & -22.493 & -1.576 \\
0.262 & 17.929 & 0 & -61.299
\end{array}\right]\left[\begin{array}{l}
\delta F_{H 1} \\
\delta F_{H 2} \\
\delta F_{C 1} \\
\delta F_{C 2}
\end{array}\right]
\end{gathered}
$$

The model (S1) was solved and eleven control structures were found, all of them with the RGA Number equal to zero. These control structures were separated into three groups. Group 1 corresponds to the structure for which the heat exchanger 121 is not selected as manipulation. Anagoulsy, groups 2 and 3 correspond to the heat exchangers 221 and 212 not consumed by the control structure respectively. It is worth to notice that the Effective Flexibility Index does not depend on the pairs, but on the selected subset of manipulations. Problem (P2) with one extra constraint, e.g. $q_{121}=$ $1 / 2 U A_{121}\left(d t_{121}+d t_{122}\right)$ for group 1 is solved. The results are given in Table 4 . It can be seen that only the control structure from group 2 presents an effective flexibility index (EFI) of 1.0, and is therefore effectively flexible after the control structure is implemented. In addition, these control structures have minimum interaction. Alternatively, problem (P4) was solved decreasing the value of $\varepsilon$ until the EFI was still greater than one giving rise to the same four control structures of group 2, avoiding the solution for the other groups.

Table 4. Effective flexibility index evaluation for each control structure.

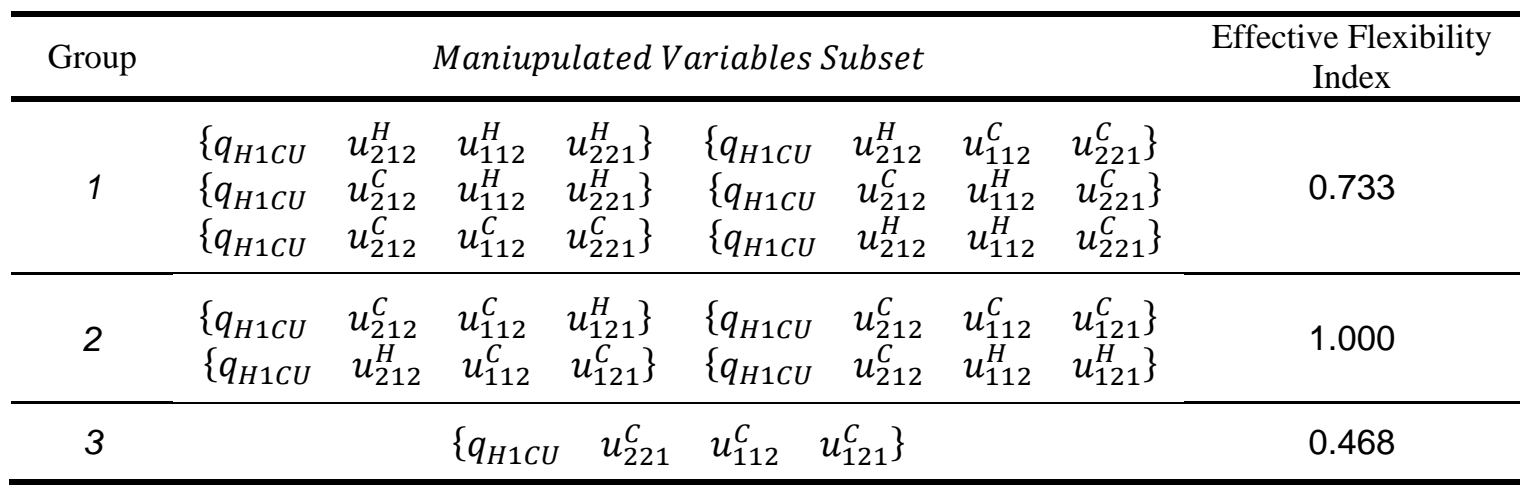


The four promising designs with control structures can be seen in Figure 10. From a steady-state point of view these designs have the same costs (TAC), and the same degree of flexibility and controllability. Since the controllability was assed solely based on the interaction, other controllability metrics may be used to characterize the structures. With this purpose, the condition number, the minimum singular value and the disturbance sensitivity were evaluated. The minimum singular value must be the largest possible, so we can avoid problems with saturation, whereas the condition number and the disturbance sensitivity must be the lowest possible. The results are presented in Table 5. It is possible to conclude that the control structure CS3 is the most promising followed by the structure CS1.

Table 5. Controllability metrics evaluation for promising control structures.

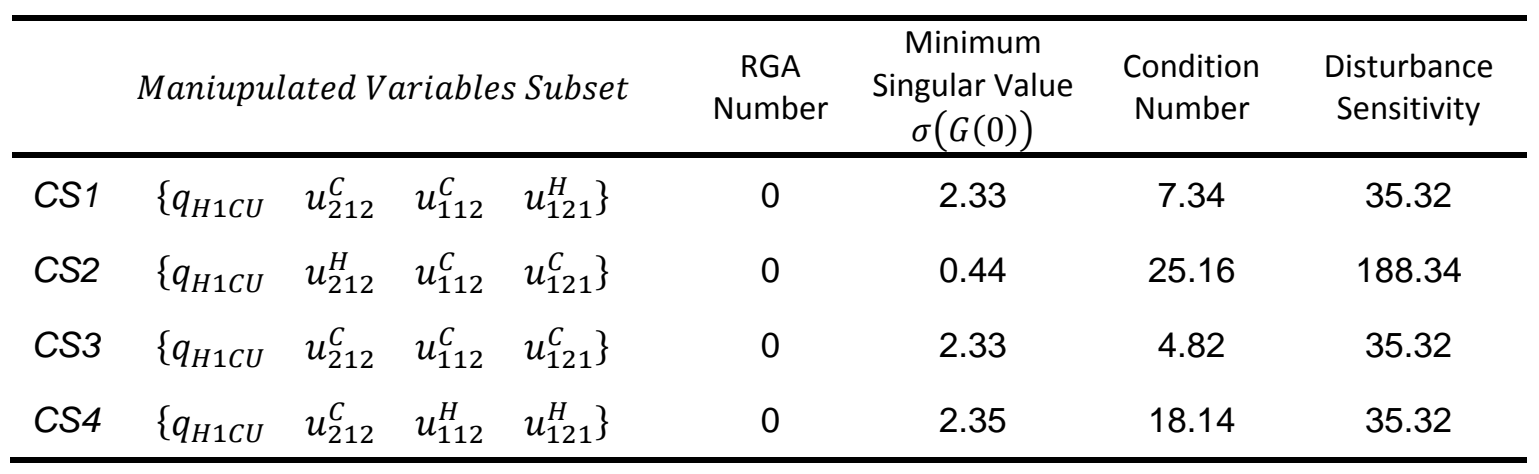

In addition, the comparison of the closed loop dynamic performance can be used as a tool for a final decision. However, the main focus here is just to show that the design can be implemented in practice. Therefore, the dynamic model and controller design were performed only for the promising structure CS3. For the dynamic model see Appendix B for a brief derivation. For each control loop, a Proportional Integral (PI) controller was designed through the model based approach proposed by Escobar and Trierweiler (2013). In this approach, for each output a closed loop performance is specified in terms of rise time and maximum overshoot allowed. A closed loop performance was selected that is two times faster than the open loop, and an overshoot of $10 \%$. The control loop design and its specifications are listed in Table 6. 


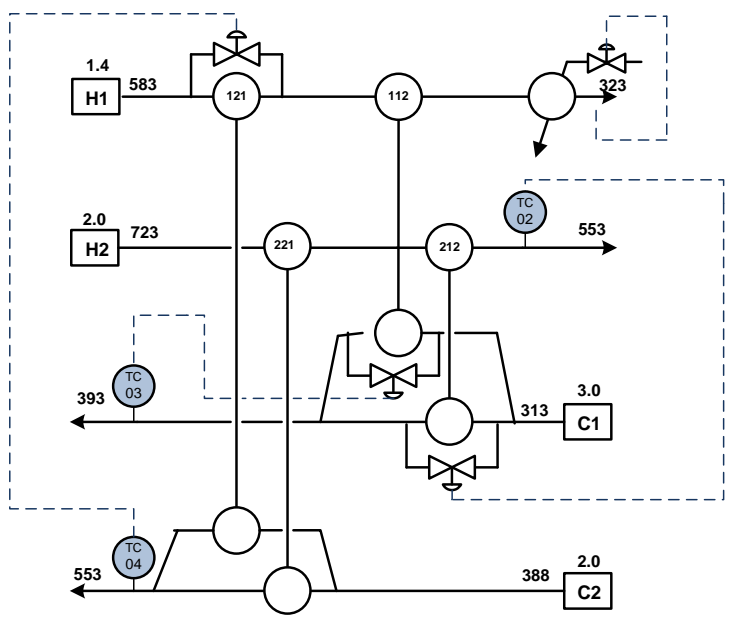

(CS1)

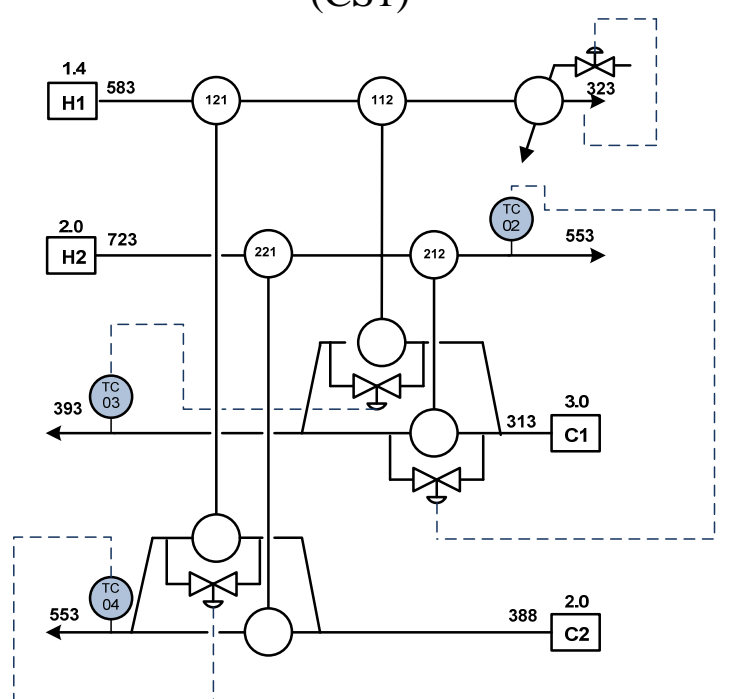

(CS3)

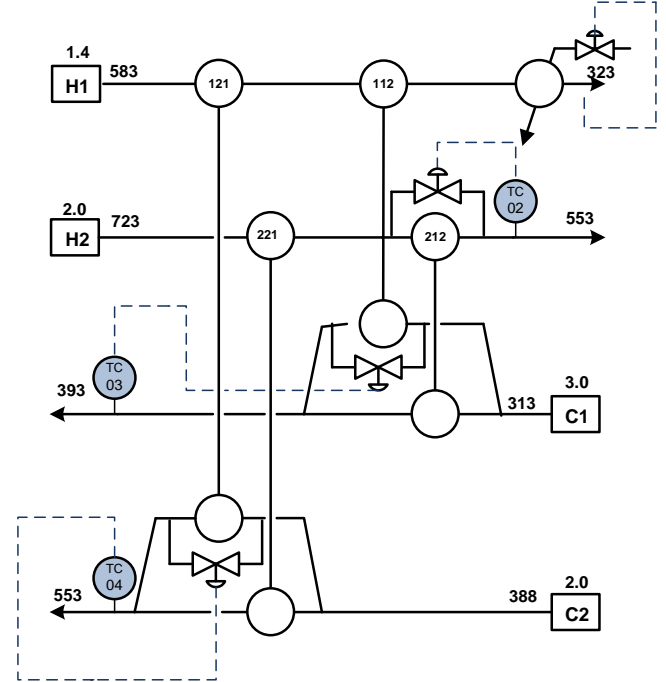

(CS2)

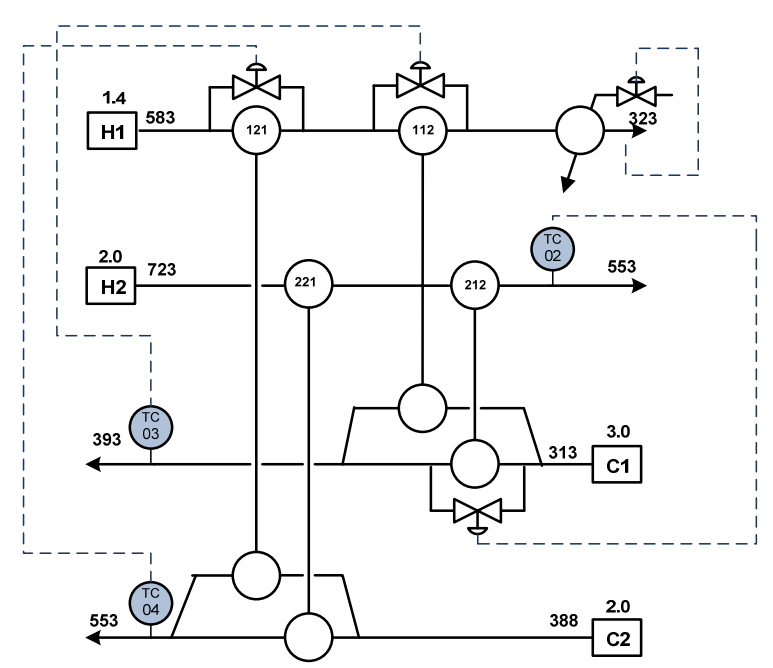

(CS4)

Figure 10. Four promising control structures for Example 1.

In order to illustrate the closed loop performance some simulations were made, the Figures are shown in Appendix C. Initially the servo response was tested, i.e. the response to a setpoint change. The setpoint change was of $1 \mathrm{~K}$ in the outlet temperature. The servo response for the control loop 1 is presented in Figure C.1. As mentioned before, when utility exchangers take place as the last exchanger, it provides a fast and direct effect on the outlet temperature and with no interaction with the other control loops. These characteristics are very interesting for the control point of view. The only issue is that an unfavorable disturbance can cause an increase in the utility consumption during the transient. However, it is assumed that if a good and fast control system is designed, the design will operate at nominal conditions most of the time during the 
operation for which the total annual cost was minimized. Another point to be considered is that since the control loop does not interact with others, it would be possible to design a controller with a response as fast as we wish. However, disturbances in other streams may also remove the outlet temperature controlled by the utility load from nominal conditions. A fast controller can implement more aggressive control actions in order to reject the disturbance. There is a trade-off between performance and robustness during the controller tuning.

Table 6. Control loop design for example 1 with CS3.

\begin{tabular}{ccccccc}
\hline $\begin{array}{c}\text { Control } \\
\text { loop }\end{array}$ & $\begin{array}{c}\text { Controlled } \\
\text { Variable } \\
(\mathrm{y})\end{array}$ & $\begin{array}{c}\text { Manipulated } \\
\text { Variable } \\
(\mathrm{u})\end{array}$ & $\begin{array}{c}\text { Setpoint } \\
\left(\mathrm{V}_{\mathrm{sp}}\right)\end{array}$ & $\begin{array}{c}\text { Manipulated } \\
\text { Nominal Value } \\
\left(\mathrm{u}_{0}\right)\end{array}$ & $\begin{array}{c}\text { Proportional } \\
\text { Gain } \\
(\mathrm{Kc})\end{array}$ & $\begin{array}{c}\text { Integral } \\
\text { time } \\
\left(\mathrm{\tau}_{\mathrm{l}}\right)\end{array}$ \\
\hline 1 & $T_{H 1}^{\text {out }}$ & $q_{H 1 C U}$ & $323 \mathrm{~K}$ & $134 \mathrm{~kW}$ & -0.054 & 6.15 \\
2 & $T_{H 2}^{\text {out }}$ & $u_{212}^{C}$ & $553 \mathrm{~K}$ & 0.4 & $-1.344 \mathrm{e}-3$ & 0.435 \\
3 & $T_{C 1}^{\text {out }}$ & $u_{112}^{C}$ & $393 \mathrm{~K}$ & 0.3 & $-3.148-3$ & 42.57 \\
4 & $T_{C 2}^{\text {out }}$ & $u_{121}^{H}$ & $553 \mathrm{~K}$ & 0.2 & $-3.456-3$ & 14.15 \\
\hline
\end{tabular}

The servo response for the control loop 2 is presented in Figure C.2. For this particular case there is a small interaction with other loops. The servo response for the control loop 3 causes a disturbance in the control loop 1. But as can be seen in Figure C.3, both loops achieve stationary conditions. In the same way, the servo response for the control loop 4, causes a disturbance in the control loop 1 and 3, but as can be seen in Figure C.4, both loops achieve stationary conditions. The regulatory response was also tested in order to check the capability of the control system to reject disturbances. At the instant zero, all the inlet temperatures were simultaneously increased by $10 \mathrm{~K}$ as a step change. The response of each control loop is shown in Figure C.5. As can be seen the network is able to reject the disturbances with this simple control structure with no saturation at the manipulations. Additional improvements in the closed loop performance can be made using different tuning parameters. However, the main idea here is only to illustrate that satisfactory performance can be reached. 


\subsection{Example 2}

The second numerical example involves two hot streams and two cold streams. The nominal data for the problem is listed in Table 7. Expected variations in the inlet temperatures of $\delta_{\mathrm{T}}=10 \mathrm{~K}$ for Hot stream H1, and $\delta_{\mathrm{T}}=5 \mathrm{~K}$ for cold stream C2, and a variation of $\delta_{\mathrm{F}}=0.4$ in the heat capacity flowrates for both streams, were assumed.

Table 7. Problem data for Example 2.

\begin{tabular}{ccccc}
\hline Stream & $\begin{array}{c}T_{\text {in }} \\
(\mathrm{K})\end{array}$ & $\begin{array}{c}T_{\text {out }} \\
(\mathrm{K})\end{array}$ & $\begin{array}{c}F \\
\left(\mathrm{~kW} \cdot \mathrm{K}^{-1}\right)\end{array}$ & $\begin{array}{c}h \\
\left(\mathrm{~kW} \mathrm{~m}^{2} \mathrm{~K}^{-1}\right)\end{array}$ \\
\hline $\mathrm{H} 1$ & $583 \pm 10$ & 323 & $1.4 \pm 0.4$ & 0.16 \\
$\mathrm{H} 2$ & 723 & 553 & 2.0 & 0.16 \\
$\mathrm{C} 1$ & 313 & 393 & 3.0 & 0.16 \\
$\mathrm{C} 2$ & $388 \pm 5$ & 553 & $2.0 \pm 0.4$ & 0.16 \\
$\mathrm{CU}$ & 303 & 323 & & 016 \\
$\mathrm{HU}$ & 573 & 573 & 0.16 \\
\hline Cost of Heat Exchangers $\left(\$ \mathrm{y}^{-1}\right)=5500+4333\left[\text { Area }\left(\mathrm{m}^{2}\right)\right]^{0.6}$ & \\
Cost of Cooling Utility $=60.576\left(\$ \mathrm{~kW}^{-1} \mathrm{y}^{-1}\right)$ \\
Cost of Heating Utility $=171.428\left(\$ \mathrm{~kW}^{-1} \mathrm{y}^{-1}\right)$
\end{tabular}

For this example, a flexible design was generated by adding critical points identified as long as the design is not flexible, solving (P1) and (P2). As presented in Table 8 the final flexible design is found at the fourth iteration with a TAC of \$148,515 per year and a Flexibility Index of 1.7134 . The critical points considered at each iteration are listed in Table 9.

Table 8. General results for flexible design for Example 2.

\begin{tabular}{ccccc}
\hline Iter. & $\begin{array}{c}\text { Operating } \\
\text { Cost }(\$ / y)\end{array}$ & $\begin{array}{c}\text { Investment } \\
\text { Cost }(\$ / y)\end{array}$ & $\begin{array}{c}\text { Total Annual } \\
\text { Cost }(\$ / y)\end{array}$ & $\begin{array}{c}\text { Flexibility } \\
\text { Index }\end{array}$ \\
\hline 1 & 24758 & 67452 & 92210 & 0.1311 \\
2 & 28823 & 96083 & 124905 & 0.1847 \\
3 & 39540 & 92563 & 132194 & 0.6358 \\
4 & 41749 & 106765 & 148515 & 1.7134 \\
\hline
\end{tabular}

The final flexible design obtained is depicted in Figure 11. In order to make the design more flexible, utility exchangers were added. For this final configuration, the steadystate model was generated and different control structures with minimum interaction were found solving problem (S1). A comparative evaluation of controllability metrics for theses control structures is presented in Table 10. It can be seen that the controllability metrics are often conflicting. For example, CS2 has a small condition number, but the higher disturbance sensitivity. In order to consider the disturbance 
sensitivity in the control structure selection, problem (S3) was solved and the solution was found to be the CS2 given in Table 10, which is also the solution found solving (P4) for simultaneously finding the CS and the effective flexibility.

Table 9. Critical points considered until each iteration.

\begin{tabular}{ccccc}
\hline Iter. & $T_{\text {in }}^{H 1}$ & $\begin{array}{c}f_{H 1} \\
(K W / K)\end{array}$ & $\begin{array}{c}T_{i n}^{C 2} \\
(K)\end{array}$ & $\begin{array}{c}f_{C 2} \\
(k W / K)\end{array}$ \\
\hline 1 & $(K)$ & 1.4 & 388 & 2.0 \\
2 & 583 & 1.8 & 383 & 2.4 \\
3 & 593 & 1.8 & 393 & 1.6 \\
4 & 593 & 1.0 & 383 & 2.4 \\
\hline
\end{tabular}

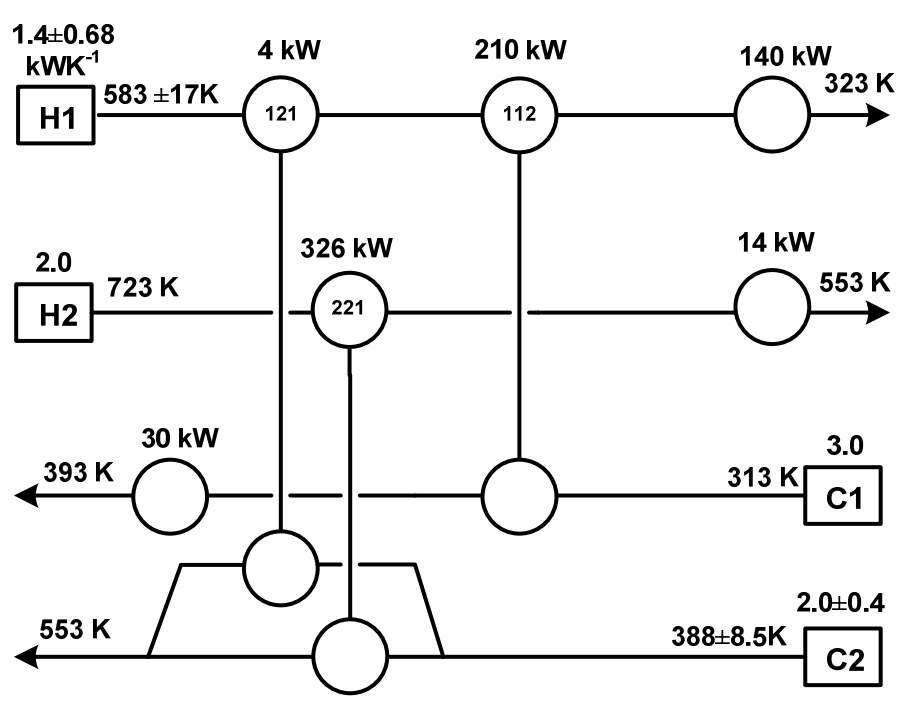

Figure 11. Flexible configuration for Example 2.

For this example, the control structure CS2 was selected for further dynamic investigation, and PI controllers were designed for each control loop. The controller design and specifications for each control loop are listed in Table 11. The servo response for the loops controlled by utility loads is presented in Figure C.6, and the servo response for the fourth control loop and its effect on the other loops, are presented in Figure C.7. It can be noted that the system has a good performance driving the system to the setpoints. The regulatory response is presented in Figure C.8. The simulation started at nominal conditions, it was implemented a step change at 250 seconds to critical point 2, according to the Table 9. After 1500 seconds, there is step change to critical point 3 , and finally at 3000 seconds a step change to critical point four in such way all critical points are considered. For all dynamic simulations, it is possible 
to notice that the control system can reject the disturbance level in a satisfactory way.

The final effective flexible design is depicted in Figure 12.

Table 10. Controllability evaluation for promising control structures.

\begin{tabular}{|c|c|c|c|c|c|c|c|c|c|}
\hline$C S$ & Maniup & lated $V$ & iriable & Subset & $\begin{array}{c}\text { RGA } \\
\text { Number }\end{array}$ & $\begin{array}{c}\text { Min } \\
\text { SingVal }\end{array}$ & $\begin{array}{c}\text { Condition } \\
\text { Number }\end{array}$ & $\begin{array}{c}\text { Disturbance } \\
\text { Sensitivity }\end{array}$ & $\begin{array}{l}\text { Effective } \\
\text { Flexibility }\end{array}$ \\
\hline 1 & $\left\{q_{H 1 C U}\right.$ & $q_{\mathrm{H} 2 \mathrm{CU}}$ & $q_{\text {HUC1 }}$ & $\left.u_{121}^{H}\right\}$ & 0 & 0.31 & 269.24 & 430.56 & 0.035 \\
\hline 2 & $\left\{q_{H 1 C U}\right.$ & $q_{\mathrm{H} 2 \mathrm{CU}}$ & $q_{\text {HUC1 }}$ & $\left.u_{221}^{H}\right\}$ & 0 & 0.33 & 202.57 & 307.17 & 0.990 \\
\hline 3 & $\left\{q_{H 1 C U}\right.$ & $q_{\mathrm{H} 2 \mathrm{CU}}$ & $q_{\text {HUC1 }}$ & $\left.u_{121}^{C}\right\}$ & 0 & 0.30 & 3.71 & 444.57 & 0.035 \\
\hline 4 & $\left\{q_{H 1 C U}\right.$ & $q_{\mathrm{H} 2 \mathrm{CU}}$ & $q_{\text {HUC1 }}$ & $\left.u_{221}^{C}\right\}$ & 0 & 0.33 & 204.24 & 346.62 & 0.990 \\
\hline
\end{tabular}

Table 11. Control loop design for example 2.

\begin{tabular}{ccccccc}
\hline $\begin{array}{c}\text { Control } \\
\text { loop }\end{array}$ & $\begin{array}{c}\text { Controlled } \\
\text { Variable } \\
(\mathrm{y})\end{array}$ & $\begin{array}{c}\text { Manipulated } \\
\text { Variable } \\
(\mathrm{u})\end{array}$ & $\begin{array}{c}\text { Setpoint } \\
\left(\mathrm{y}_{\mathrm{sp}}\right)\end{array}$ & $\begin{array}{c}\text { Manipulated } \\
\text { Nominal Value } \\
\left(\mathrm{u}_{0}\right)\end{array}$ & $\begin{array}{c}\text { Proportional } \\
\text { Gain } \\
(\mathrm{Kc})\end{array}$ & $\begin{array}{c}\text { Integral } \\
\text { time } \\
\left(\mathrm{\tau}_{1}\right)\end{array}$ \\
\hline 1 & $T_{H 1}^{\text {out }}$ & $q_{H 1 C U}$ & $323 \mathrm{~K}$ & $140 \mathrm{~kW}$ & -0.185 & 21.14 \\
2 & $T_{H 2}^{\text {out }}$ & $q_{H 2 C U}$ & $553 \mathrm{~K}$ & $14 \mathrm{~kW}$ & -0.190 & 2.196 \\
3 & $T_{C 1}^{\text {out }}$ & $q_{H U C 1}$ & $393 \mathrm{~K}$ & $30 \mathrm{~kW}$ & 0.301 & 1.043 \\
4 & $T_{C 2}^{\text {out }}$ & $u_{221}^{H}$ & $553 \mathrm{~K}$ & 0.35 & $-3.456-3$ & 34.28 \\
\hline
\end{tabular}

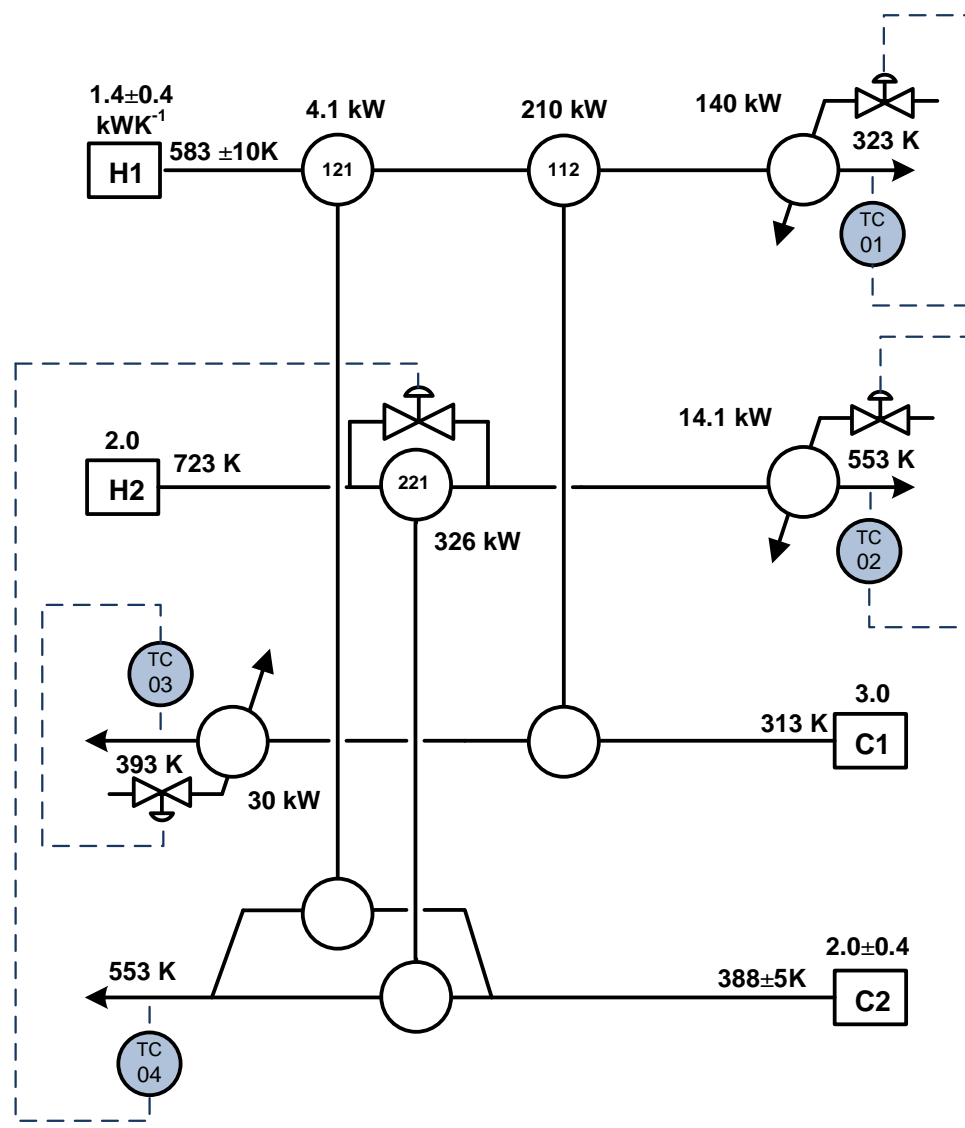

Figure 12. Final effective flexible design for Example 2. 


\subsection{Example 3}

The third numerical example involves six hot streams and one cold stream. The nominal and economic data for the problem is presented in Table 12. Expected variations in the inlet temperatures of $\delta_{\mathrm{T}}=10 \mathrm{~K}$ and a variation of $\delta_{\mathrm{F}}=5 \%$ in the heat capacity flowrates, were assumed. The minimum number of stages in the superstructure was set to 3. The first design was generated with nominal condition. The flexibility index was 1.22 pointing out the flexible design. However, solving the problem (P4) even for large upper bounds on the controllability metric, the maximum effective flexibility index was 0.835. The critical point identified was all inlet temperatures down by $-10 \mathrm{~K}$, the hot flowrates increased by $5 \%$ and the cold flowrates decreased by $5 \%$. The second design was obtained solving (P1) resulting in a design with a TAC of \$ 975,959 per year and a FI of 1.89, for which a feasible control structure was found with effective flexibility of 1.16. The general results are summarized in Table13.

Table 12. Problem data for Example 3.

\begin{tabular}{ccccc}
\hline Stream & $\begin{array}{c}T_{\text {in }} \\
(\mathrm{K})\end{array}$ & $\begin{array}{c}T_{\text {out }} \\
(\mathrm{K})\end{array}$ & $\begin{array}{c}F \\
\left(\mathrm{~kW} \cdot \mathrm{K}^{-1}\right)\end{array}$ & $\begin{array}{c}h \\
\left(\mathrm{~kW} \mathrm{~m}^{2} \mathrm{~K}^{-1}\right)\end{array}$ \\
\hline H1 & $630 \pm 10$ & 460 & $9.0 \pm 5 \%$ & 1.2 \\
H2 & $550 \pm 10$ & 480 & $6.5 \pm 5 \%$ & 0.6 \\
H3 & $530 \pm 10$ & 480 & $3.0 \pm 5 \%$ & 0.4 \\
H4 & $470 \pm 10$ & 400 & $36.0 \pm 5 \%$ & 0.6 \\
H5 & $450 \pm 10$ & 310 & $7.0 \pm 5 \%$ & 0.4 \\
H6 & $410 \pm 10$ & 350 & $72.0 \pm 5 \%$ & 0.4 \\
C1 & $310 \pm 10$ & 650 & $27.0 \pm 5 \%$ & 1.2 \\
CU & 300 & 330 & & 1.2 \\
HU & 700 & 700 & 0.3 \\
\hline Cost of Heat Exchangers $\left(\$ \mathrm{y}^{-1}\right)=100+866\left[\text { Area }\left(\mathrm{m}^{2}\right)\right]^{0.6}$ & \\
Cost of Cooling Utility $=52\left(\$ \mathrm{~kW}^{-1} \mathrm{y}^{-1}\right)$ \\
Cost of Heating Utility $=176\left(\$ \mathrm{~kW}^{-1} \mathrm{y}^{-1}\right)$ \\
\hline
\end{tabular}

Table 13. General results for flexible and controllable design for example 3.

\begin{tabular}{cccccc}
\hline Iter. & $\begin{array}{c}\text { Operating } \\
\text { Cost }(\$ / y)\end{array}$ & $\begin{array}{c}\text { Investment } \\
\text { Cost }(\$ / y)\end{array}$ & $\begin{array}{c}\text { Total Annual } \\
\text { Cost }(\$ / y)\end{array}$ & $\begin{array}{c}\text { Flexibility } \\
\text { Index }\end{array}$ & $\begin{array}{c}\text { Effevtive } \\
\text { Flexibility }\end{array}$ \\
\hline 1 & 755670.8 & 115079.5 & 870750.3 & 1.22 & 0.835 \\
2 & 794478.8 & 181480.8 & 975959.8 & 1.89 & 1.16 \\
\hline
\end{tabular}

The final design with best control structure is depicted in Figure 13. For this example servo responses to a unit change are shown only for the first and the last control loop in 
Figures C.9 and Figure C.10, respectively. Additional simulations for the servo response were suppressed. In general, good performance was obtained. Finally, in Figure C.11 shows the regulatory response for a change from the nominal conditions to the critical point. The results show the disturbance rejection. Thus, it is possible to conclude that the design is flexible and controllable using the proposed approach.

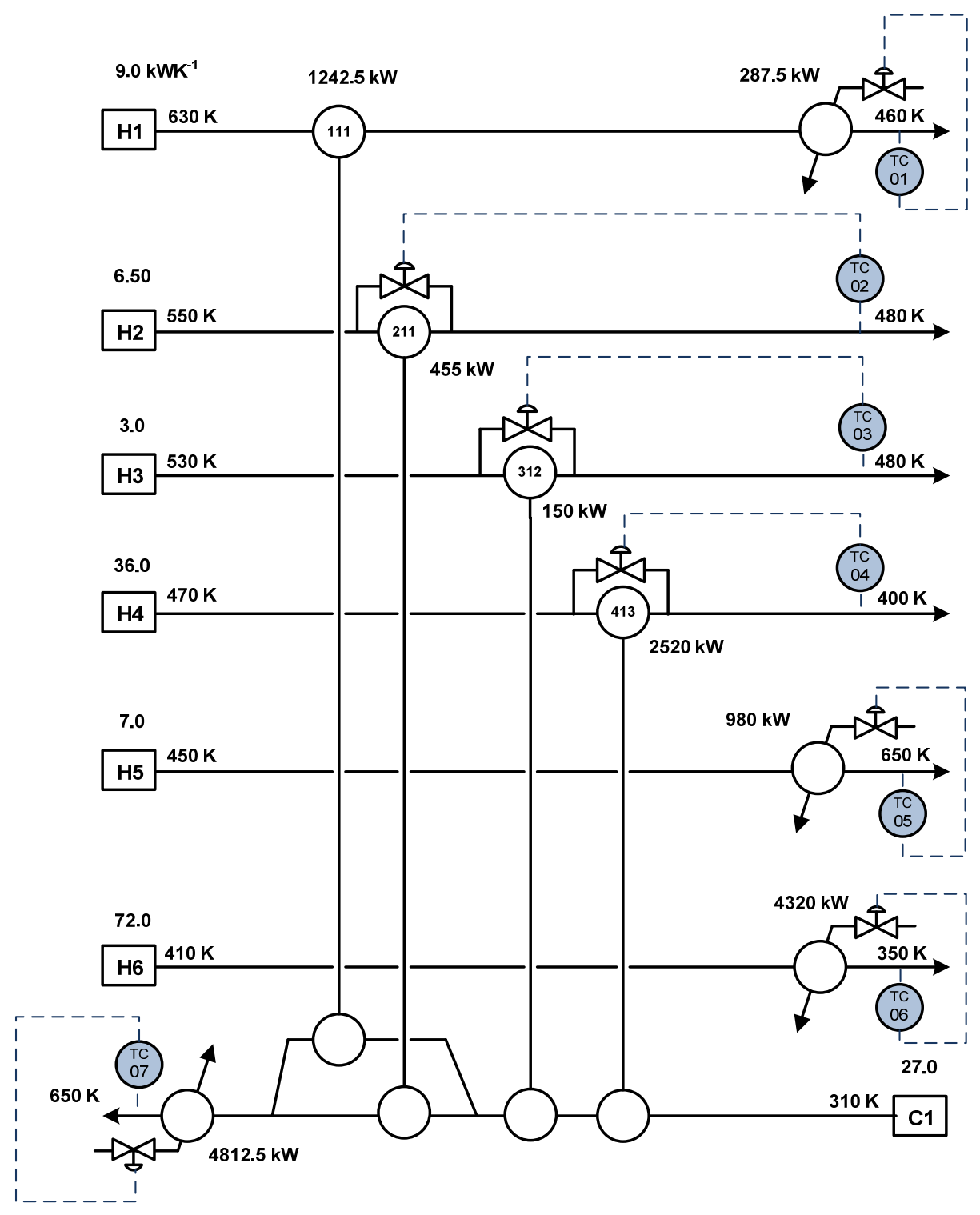

Figure 13. Final configuration and control structure for the Example 3.

\section{Conclusions}

In this work the synthesis of heat exchanger networks with operability considerations has been addressed. A conceptual framework was developed to incorporate both flexibility and controllability aspects in synthesis. The proposed approach is based on 
the two-stage strategy for flexible designs. The control system was considered during the operating stage. Using a steady-state transfer function model, promising control structures were found solving an MILP subproblem, which was coupled with the flexibility analysis in order to obtain a network that is not only optimally designed in terms of costs, but also exhibits good operability properties. The control structure and the effective flexibility may be solved either sequentially or simultaneously. Once the design is statically flexible and controllable, using the dynamic model the controllers can be designed and the dynamic closed loop performance can be checked. Three examples were used to illustrate the whole procedure, and used to show the suitability of the proposed approach.

The main limitation of the proposed approach is that the controllability analysis is performed using steady-state and linear models, which may result in significant deviations from the nonlinear model, and restricts its applicability. However, given the combinatorial nature of HENS, the application of dynamic nonlinear models is a very challenging problem. The dimension of the differential algebraic equation (DAE) system increases drastically for even medium size problems, resulting in a MIDO problem that is virtually intractable. However, this problem is significantly simplified if we restrict the analysis to the steady-state. This limitation may be circumvented with either better controller tuning, or some static overdesign if some constraint is violated by the control profiles during the dynamic analysis. In addition, the multiperiod problem (P1) and the effective flexibility index (P4) have a block diagonal structure, which can be exploited by specialized decomposition techniques such as Lagrangean decomposition. In this way, the proposed approach can be enhanced for its application to large scale problems. 


\section{Nomenclature}

\section{Indices}

\begin{tabular}{ll}
\hline$i$ & hot stream \\
$j$ & cold stream \\
$k$ & superstructure stage \\
$p e r i o d$ & heat exchanger \\
$k x$ & \\
Sets & \\
\hline$C P$ & set of cold process stream $j$ \\
$H P$ & set of hot process stream $i$ \\
$H E$ & set of heat exchangers \\
$M V$ & set of potential manipulated variables \\
$D V$ & set of disturbance variables \\
$P R$ & set of periods
\end{tabular}

\section{Variables}

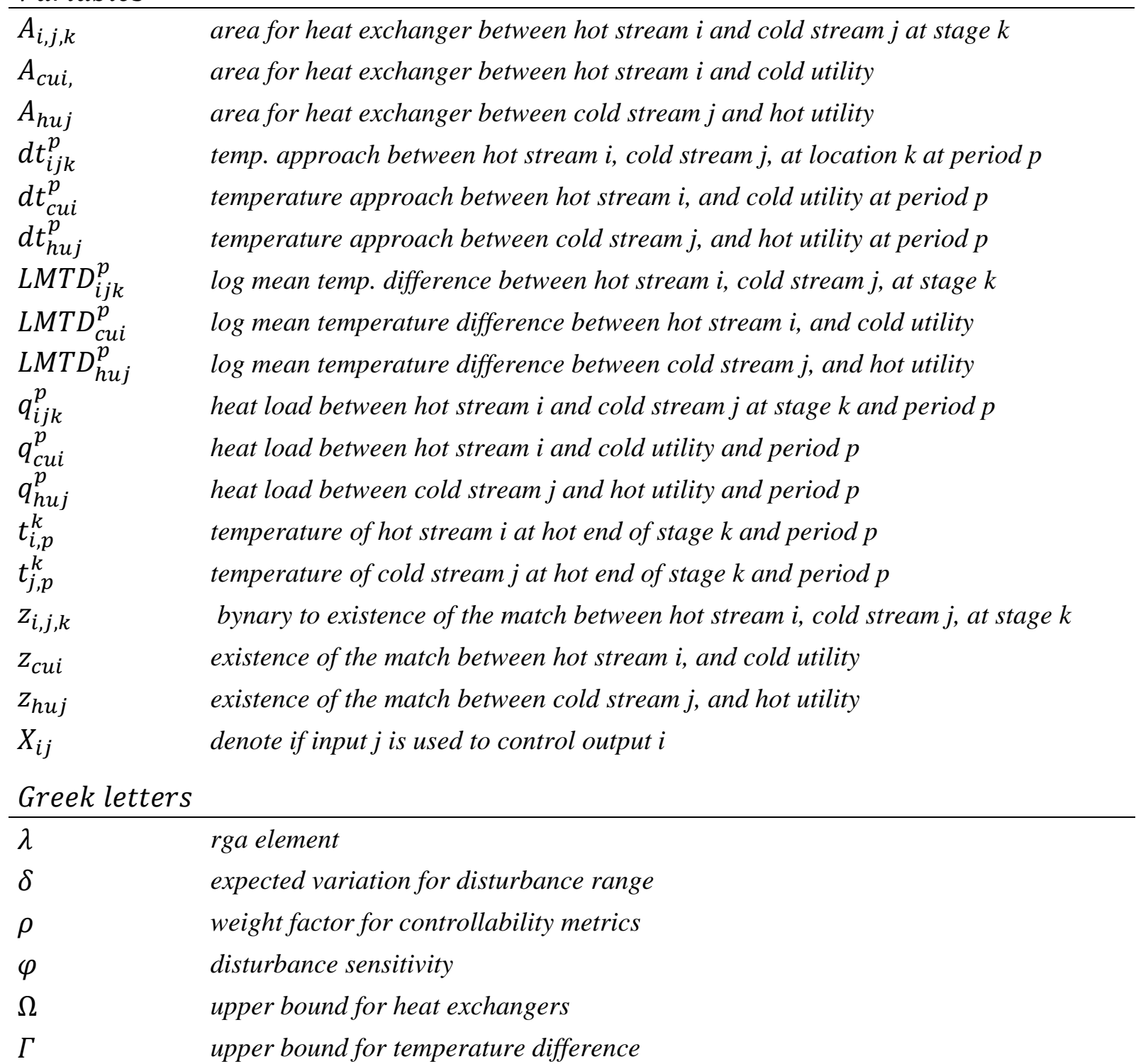




\section{References}

Aaltola J. Simultaneous Synthesis of Flexible Heat Exchanger Networks. Department of Mechanical Engineering, Helsinki University of Technology, Finland. Ph.D. Thesis, 2002.

Aguilera N, Marchetti JL. Optimizing and controlling the operation of heat exchanger Networks. AIChE Journal 1998; 44(5), 1090-1104.

Biegler LT, Grossmann IE, Westerberg, WA. Systematic Methods of Chemical Process Design. Prentice Hall International Series in the Physical and Chemical Engineering Sciences, 1997.

Björk KM, Westerlund T. Global optimization of heat exchanger network synthesis problems with and without the isothermal mixing assumption. Computers \& Chemical Engineering 2002; 26(11):1581-1593.

Bristol E. On a new measure of interaction for multivariable process control. In: IEEE Transaction on Automatic Control 1966; 11(1):133-134.

Chen JJJ. Comments on improvements on a replacement for the logarithmic mean. Chemical Engineering Science 1987; 21:775-780.

Chen LC, Hung PS. Simultaneous synthesis of flexible heat-exchange networks with uncertain sourcestream temperatures and flow rates. Industrial \& Engineering Chemistry Research 2004; 43:5916-5928.

Chen LC, Hung PS. Synthesis of flexible heat exchange networks and mass exchange network. Computers \& Chemical Engineering 2007; 31:1619-1632.

Ciric AR, Floudas CA (1991). Heat Exchanger Network Synthesis without Decomposition. Computers \& Chemical Engineering 1991; 15(6):385-396.

Escobar M, Trierweiler JO. Operational Controllability of Heat Exchanger Networks. In: Proceedings of 9th International Symposium on Dynamics and Control of Process Systems, Leuven, 2010.

Escobar M, Trierweiler JO, Grossmann IE. SynFlex: A computational Framework for synthesis of Flexible Heat Exchanger Networks. Computer Aided Chemical Engineering 2011; 32(2):2276-2290.

Escobar M, Trierweiler JO. Optimal heat exchanger network synthesis: A case study comparison. Applied Thermal Engineering, 2013, 51:801-826

Escobar M, Trierweiler JO. Multivariable PID Controller Design for Chemical Processes by Frequency Response Approximation. Chemical Engineering Science, 2013, doi 10.1016/j.ces.2012.11.011, in press.

Floudas CA, Ciric AR, Grossmann IE. Automatic Synthesis of Optimum Heat Exchanger Network Configurations. AIChE Journal 1986; 32 (2):276-290.

Floudas CA, Grossmann IE. Synthesis of Flexible Heat Exchanger Networks with Uncertain Flowrates and Temperatures. Computers \& Chemical Engineering 1987; 11:319-336.

Furman KC, Sahinidis N. A Critical Review and Annotated Bibliography for Heat Exchanger Network Synthesis in the 20th Century. Industrial \& Engineering Chemistry Research 2002; 41(10): 2335-2370.

Glemmestad B. Optimal Operation of Integrated Processes. Department of Process Technology, Trondheim, Norwegian University of Science and Technology. Ph.D. Thesis, 1997.

Gonzalez AH, Odloak D, Marchetti JL. Predictive control applied to heat-exchanger networks. Chemical Engineering Processing 2006; 45, 661-671.

Grossmann IE, Floudas CA. Active constraint strategy for flexibility analysis in chemical processes. Computers \& Chemical Engineering 1987; 11:675-693.

Halemane P, Grossmann IE. Optimal process design under uncertainty. AIChE Journal 1983; 29:425433.

Lersbamrungsuk V, Srinophakun T, Narashimhan, Skogestad S. Control structure selection for Optimal Operation of Heat Exchanger Networks. AIChE Journal 2008; 54(1):150-162.

Ponce-Ortega JM, Jiménez-Gutiérrez A, Grossmann IE. Optimal synthesis of heat exchanger networks involving isothermal process streams. Computers \& Chemical Engineering 2008; 32(8):1918-1942. 
Kookos IK, Perkins JD. An Algorithm for Simultaneous Process Design and Control. Industrial \& Engineering Chemistry Research 2001; 40:4079-4088.

Konukman AES, Çamurdan MC, Akman U. Simultaneous flexibility targeting and synthesis of minimum-utility heat-exchanger networks with superstructure-based MILP formulation. Chemical Engineering and Processing 2002; 41:501-518.

Kotjabasakis E, Linnhoff B. Sensitivity Tables or the Design of Flexible Processes. How much Contingency in Heat Exchanger Networks is Cost-Effective? Chemical Engineering Research 1986; 64, 199-211.

Linnhoff B, Hindmarsh E (1983). The Pinch Design Method for Heat Exchanger Networks. Chemical Engineering Science 1983; 38(5): 745-763.

López-Maldonado LA, Ponce-Ortega JM, Segovia-Hernández JG. Multiobjective synthesis of heat exchanger networks minimizing the total annual cost and the environmental impact. Applied Thermal Engineering 2011; 31:1099-1113.

Ma K, Hui C, Yee TF. Constant approach temperature model for HEN retrofit. Applied Thermal Engineering 2000; 20(15):1505-1533.

Marselle DF, Morari M, Rudd DF. Design of Resilient Processing Plants - II. Design and Control of Energy Management Systems. Chemical Engineering Science 1982; 37, 259-270.

Mathisen KW. Integrated Design and Control of Heat Exchanger Networks. In: Trondheim University NTH, Norway. Ph.D. Thesis, 1994.

Mizutani FT, Pessoa FLP, Queiroz EM, Hauan S, Grossmann IE. Mathematical programming model for heat exchanger network synthesis including detailed heat exchanger designs. 2. Network synthesis. Industrial \& Engineering Chemistry Research 2003; 42:4019-4027.

Papalexandri KP, Pistikopoulos EN. Synthesis and Retrofit Design of Operable Heat Exchanger Networks. 1. Flexibility and Structural Controllability Aspects. Industrial and Engineering Chemistry Research, 1994; 33(7).

Papoulias SA, Grossmann IE. A Structural Optimization Approach in Process Synthesis II. Heat Recovery Networks. Computers \& Chemical Engineering 1983; 7(6):707.

Ravagnani MA, Caballero JA. Optimal heat exchanger network synthesis with the detailed heat transfer equipment design. Computers \& Chemical Engineering 2007; 31(11):1432-144.

Rooney WC, Biegler LT. Optimal Process Design with Model Parameter Uncertainty and Process Variability. AIChE Journal 2003; 49:438-449.

Saboo AK, Morari M, Woodcock DC. Design of Resilient Processing Plants - VIII A Resilience Index for Heat Exchanger Networks. Chemical Engineering Science 1985; 40 (8), 1553-1565.

Skogestad S, Postlethwaite I. Multivariable Feedback Control, Analysis and Design. John Wiley \& Sons, UK, 1996.

Soršak A, Kravanja Z. MINLP retrofit of heat exchanger networks comprising different exchanger types . Computer Aided Chemical Engineering 2002; 10:349-354.

Swaney RE, Grossmann IE. An index for operational flexibility in chemical process design - Part I: Formulation and theory. AIChE Journal 1985; 26:139.

Uzturk D, Akman U. Centralized and decentralized control of retrofit heat-exchanger networks. Computers \& Chemical Engineering 1997; 21, Suppl., 373-378.

Verheyen W, Zhang N. Design of flexible heat exchanger network for multi-period operation. Chemical Engineering Science 2006; 61:7730-7753.

Wal M, Jager B. A review of methods for input/output selection. Automatica 2001; 37(4):487-510.

Yan QZ, Yang YH, Huang YL. Cost-Effective Bypass Design of Highly Controllable Heat-Exchanger Networks. AIChE Journal 2001; 47(10):2253-2275.

Yee TF, Grossmann IE. Simultaneous Optimization Models for Heat Integrations II. Heat Exchanger Network Synthesis. Computers \& Chemical Engineering 1990; 14(10):1165. 


\section{LIST OF CAPTIONS}

Figure 1. An overall process system.

Figure 2. Possible manipulated inputs in HENs.

Figure 3. Two-stage strategy for optimal design under uncertainty.

Figure 4. Outline of proposed strategy for flexible and controllable design.

Figure 5. Superstructure for SYNHEAT model (Yee and Grossman, 1990) exemplified for two hot streams, to cold streams and two stages.

Figure 6. Outline of the solution strategy.

Figure 7. Nominal Design for numerical Example 1.

Figure 8. Two possible Control Structures for Nominal Design.

Figure 9. Flexible Configuration for Example 1.

Figure 10. Four promising control structures for Example 1.

Figure 11. Flexible configuration for Example 2.

Figure 12. Final effective flexible design for Example 3.

Figure 13. Final configuration and control structure for Example 3.

Figure B.1. General heat exchanger with bypasses.

Figure B.2: Sketch of mixing tank model.

Figure C.1. Servo Response for control loop 1.

Figure C.2. Servo Response for control loop 2.

Figure C.5. Regulatory response for all control loops - example 1.

Figure C.6. Servo response for the loops controlled by utility loads - example 2.

Figure C.7. Servo response for control loop 4 and effect on control loop 2 - example 2.

Figure C.8. Regulatory response for all control loops -example 2.

Figure C.9. Servo response for control loop 1 - example 3.

Figure C.9. Servo response for control loop 1 - example 3.

Figure C.10. Servo response for control loop 7 and effect on control loop 1,2,3 and 4.

Figure C.11. Regulatory response for all control loops - example 3.

Table 1. Problem data for Example 1.

Table 2. General Results for TSS applied to Example 1.

Table 3. Uncertain Parameters for the Points considered.

Table 4. Controllability Evaluation for promising control structures.

Table 5. Controllability metrics evaluation for promising control structures.

Table 2. Control loop design for example 1 with CS3.

Table 7. Problem data for Example 2.

Table 8. General results for flexible design for Example 2.

Table 3. Critical points considered until each iteration.

Table 4. Controllability evaluation for promising control structures.

Table 11. Control loop design for Example 2.

Table 12. Problem data for Example 3. 
Table 13. General results for flexible and controllable design for example 3.

\section{Appendix A. Multiperiod Optimization Model for HENS}

$$
\begin{aligned}
& \min _{\substack{x \in \Omega=\\
\Omega_{\mathrm{N}} \cup \Omega_{\mathrm{L}}}}\left\{\begin{array}{c}
\sum_{p \in P R} \frac{D O P(p)}{N_{P}}\left(\sum_{i \in H P} c_{c u} q_{c u i}^{p}+\sum_{j \in C P} c_{h u} q_{h u j}^{p}\right)+\sum_{i \in H P} \sum_{j \in C P} \sum_{k \in S T} c z_{i j k}+\sum_{i \in H P} c z_{c u i}+\sum_{j \in C P} c z_{h u j} \\
\sum_{i \in H P} \sum_{j \in C P} \sum_{k \in S T} c_{i j}\left(A_{i j k}\right)^{\beta}+\sum_{i \in H P} c_{c u i}\left(A_{c u i}\right)^{\beta}+\sum_{j \in C P} c_{h u j}\left(A_{h u j}\right)^{\beta}
\end{array}\right\} \\
& x=\left\{z_{i j k}, z_{c u i}, z_{h u j}, t_{i k}^{p}, t_{j k}^{p} ; d t_{i j k}^{p}, d t_{c u i}^{p}, d t_{h u j}^{p} ; q_{i j k}^{p}, q_{c u i}^{p}, q_{h u j}^{p} i \in H P, j \in C P, k \in S T, p \in P R\right\} \\
& \Omega_{N}=\left\{\begin{array}{c}
A_{i j k} \geq q_{i j k}^{p}\left(h_{i}^{-1}+h_{j}^{-1}\right) /\left[d t_{i j k}^{p} d t_{i j k+1}^{p} \frac{d t_{i j k}^{p}+d t_{i j k+1}^{p}}{2}\right]^{1 / 3} \\
A_{c u i} \geq q_{c u i}^{p}\left(h_{i}^{-1}+h_{C U}^{-1}\right) /\left[d t_{c u i}^{p}\left(T_{i}^{o u t}-T_{C U}^{i n}\right) \frac{d t_{c u i}^{p}+\left(T_{i}^{o u t}-T_{C U}^{i n}\right)}{2}\right]^{1 / 3} \\
A_{h u j} \geq q_{h u j}\left(h_{j}^{-1}+h_{H U}^{-1}\right) /\left[d t_{h u j}^{p}\left(T_{H U}^{i n}-T_{j}^{\text {out }}\right) \frac{d t_{h u j}^{p}+\left(T_{H U}^{i n}-T_{j}^{o u t}\right)}{2}\right]^{1 / 3}
\end{array}\right\}
\end{aligned}
$$




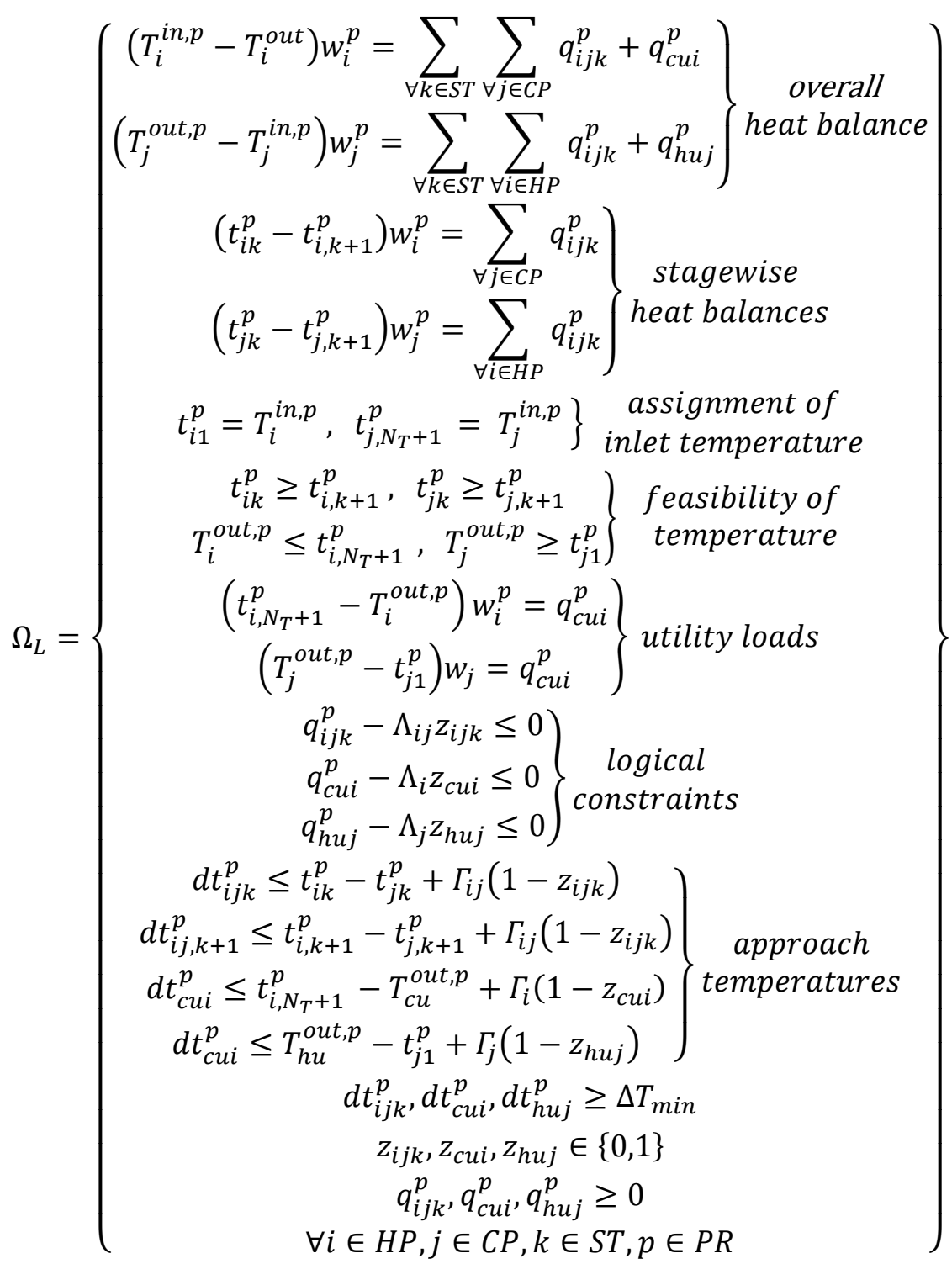

\section{Appendix B. Static and Dynamic Model for HEN}

\section{B.1 Static Modeling}

The steady-state transfer function model is obtained based on the unit model for each heat exchanger. A general heat exchanger with bypass on hot and cold side is sketched in Figure B.1. 


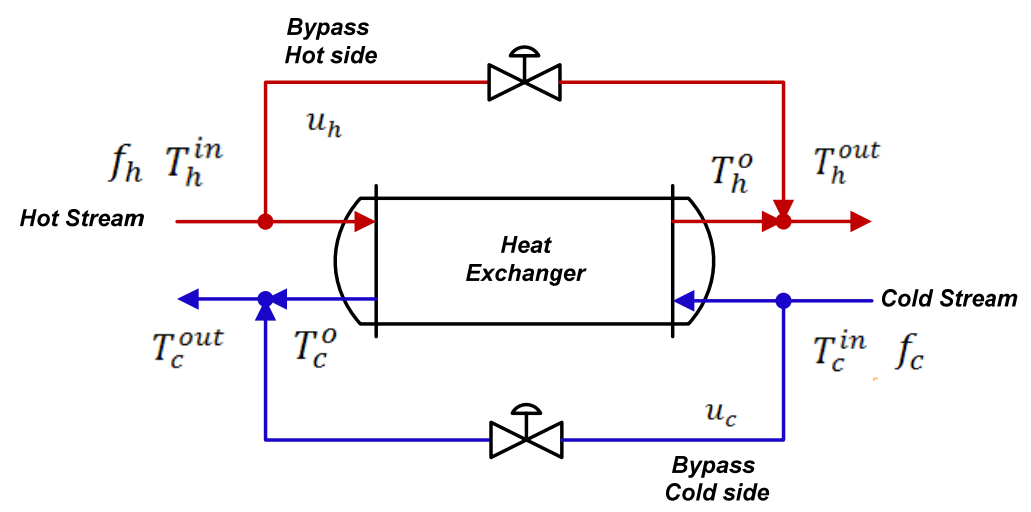

Figure B.1. General heat exchanger with bypasses.

where $f$ the heat capacity flowrate; $T$ the temperature; $U$ the overall heat transfer; $A$ the Area and $L M T D$ the logarithmic mean temperature difference.

Model A: Unit Model for the system Heat Exchanger with Bypasses

$$
\begin{aligned}
& {\left[\begin{array}{l}
T_{h}^{\text {out }} \\
T_{c}^{\text {out }}
\end{array}\right]=\left[\begin{array}{cc}
1-u_{h} & 0 \\
0 & 1-u_{c}
\end{array}\right]\left[\begin{array}{l}
T_{h}^{o} \\
T_{c}^{o}
\end{array}\right]+\left[\begin{array}{cc}
u_{h} & 0 \\
0 & u_{c}
\end{array}\right]\left[\begin{array}{c}
T_{h}^{\text {in }} \\
T_{c}^{\text {in }}
\end{array}\right]} \\
& {\left[\begin{array}{l}
T_{h}^{o} \\
T_{c}^{o}
\end{array}\right]=\frac{1}{\left(R_{h}^{\prime}-a^{\prime}\right)}\left[\begin{array}{cc}
R_{h}^{\prime}-1 & 1-a^{\prime} \\
R_{h}^{\prime}\left(1-a^{\prime}\right) & a^{\prime}\left(R_{h}^{\prime}-1\right)
\end{array}\right]\left[\begin{array}{c}
T_{h}^{\text {in }} \\
T_{c}^{\text {in }}
\end{array}\right]}
\end{aligned}
$$

Ratio between effective heat capacity flow rates:

$$
R_{h}^{\prime} \equiv \frac{\left(1-u_{h}\right) f_{h}}{\left(1-u_{c}\right) f_{c}}=\frac{T_{c}^{o}-T_{c}^{i n}}{T_{h}^{i n}-T_{h}^{o}}
$$

Ratio between terminal temperature differences:

$$
a^{\prime} \equiv \frac{T_{h}^{i n}-T_{c}^{o}}{T_{h}^{o}-T_{c}^{i n}}=\exp \left(\frac{U^{\prime} A}{\left(1-u_{h}\right) f_{h}}\left(1-R_{h}^{\prime}\right)\right)
$$

Corrected Global Heat Transfer Coefficient:

$$
\frac{1}{U^{\prime}}=\frac{1}{h_{h}\left(1-u_{h}\right)}+\frac{1}{h_{c}\left(1-u_{c}\right)}
$$

The nonlinear model is linearized, i.e. differentiated in respect to the inlet temperatures $\left(T^{i n}\right)$, flowrates $(f)$ and bypass fractions $(u)$, using Taylor series expansion, neglecting high-order differentiation terms. Based on structural information from the solution of (P1) and algebraic manipulation it was developed a routine in Matlab for the generation of the steady-state model. For more details about this algebraic manipulation, see Escobar and Trierweiler (2010). 


\section{B.2 Dynamic Modeling}

. The dynamic model is based on the hypothesis that a heat exchanger dynamic behavior can be described by a series of $N$ tanks depicted in Figure B.2.

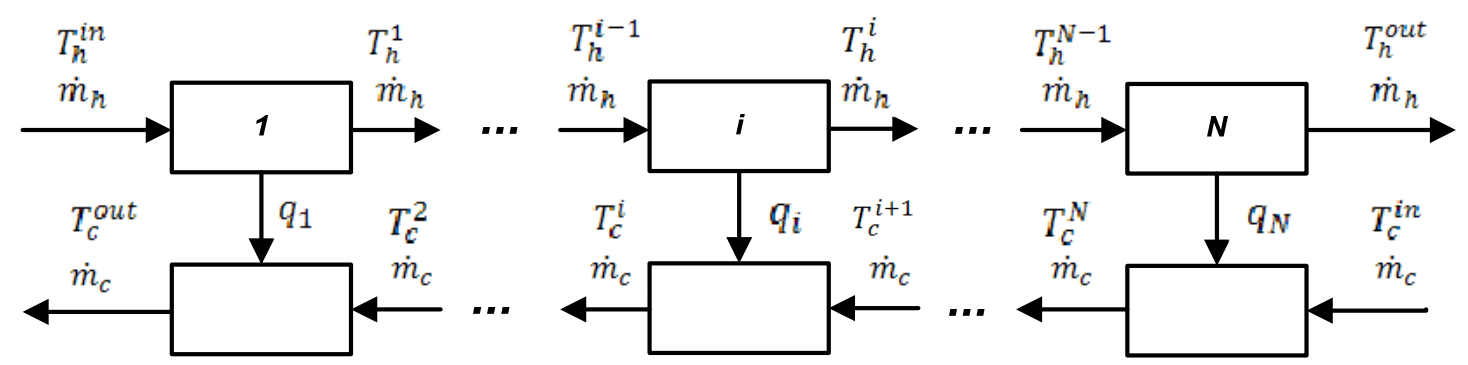

Figure B.2: Sketch of mixing tank model.

The dynamic model for each stage can be described by the following equations:

$$
\begin{gathered}
\rho_{h} V_{h}^{i} C p_{h} \frac{d T_{h}^{i}}{d t}=\dot{m}_{h} C p_{h}\left(T_{h}^{i-1}-T_{h}^{i}\right)-U A^{i} \Delta T_{e f}^{i} \\
\rho_{c} V_{c}^{i} C p_{c} \frac{d T_{c}^{i}}{d t}=\dot{m}_{c} C p_{c}\left(T_{c}^{i+1}-T_{c}^{i}\right)+U A^{i} \Delta T_{e f}^{i} \\
\Delta T_{e f}^{i}=\frac{\left(T_{h}^{i-1}-T_{c}^{i}\right)+\left(T_{h}^{i}-T_{c}^{i+1}\right)}{2}
\end{gathered}
$$

The effective temperature driving force for each cell was represented by the arithmetic mean temperature difference. The tanks are assumed to be equally sized, and the fluid has the same physical properties of water. The volume of each tank is determined by assuming a constant proportionality of $V / A=8.3 \mathrm{e}-4 \mathrm{~m}^{3} / \mathrm{m}^{2}$. In general, four tanks $(N=4)$ as indicated by Glemmestad (1997) provide a good representation.

\section{Appendix C. Closed Loop Simulations}



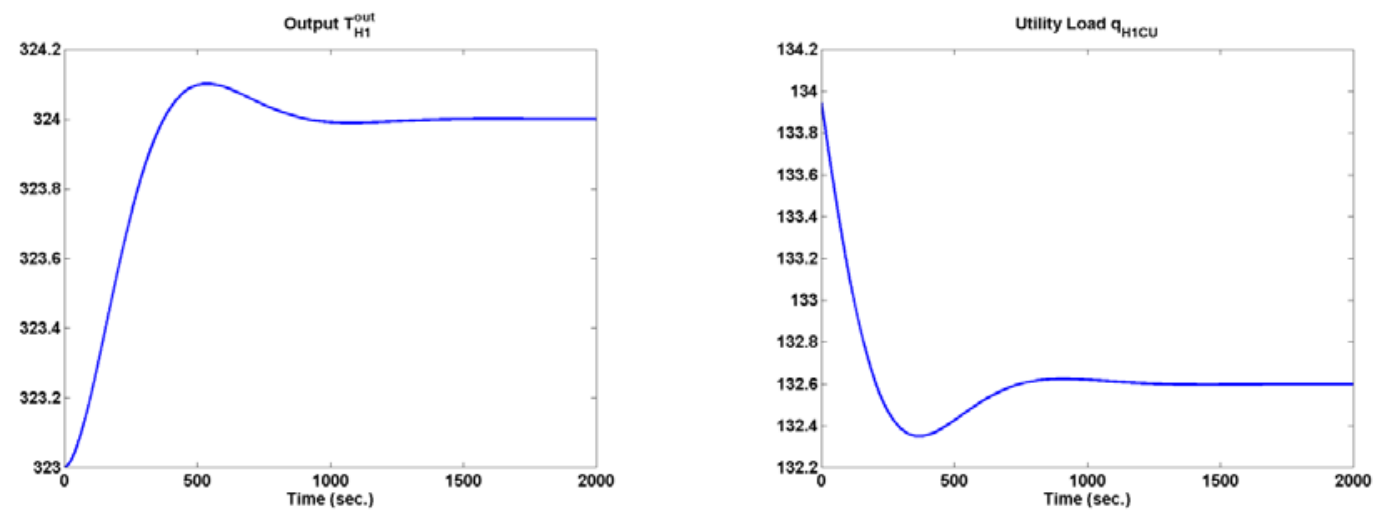

Figure C.1. Servo Response for control loop 1 -example 1.
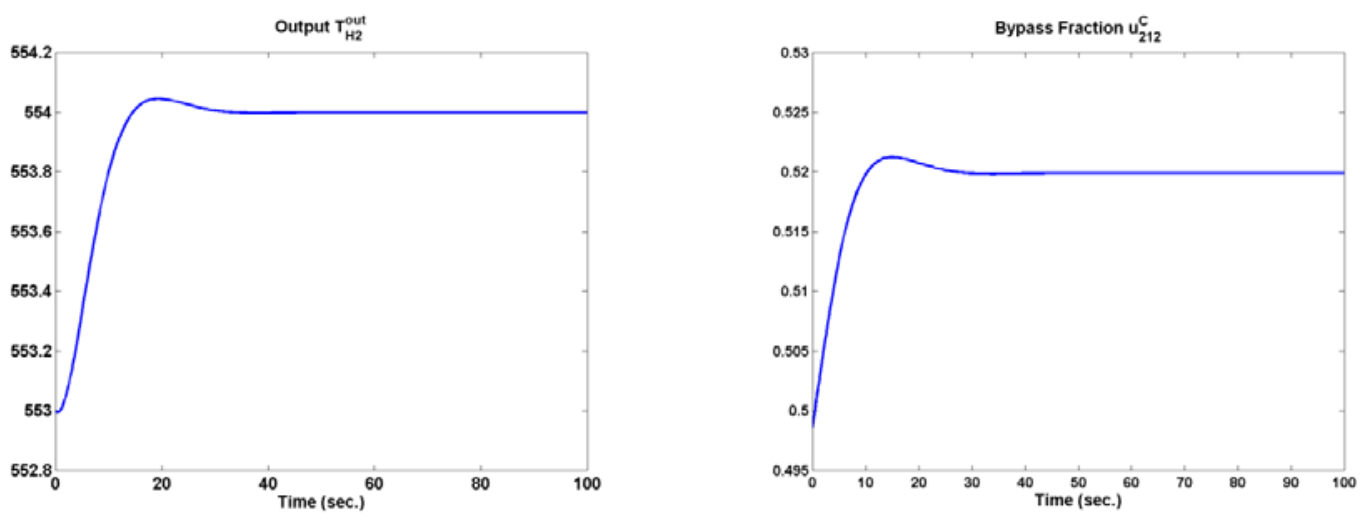

Figure C.2. Servo Response for control loop 2 - example 1.
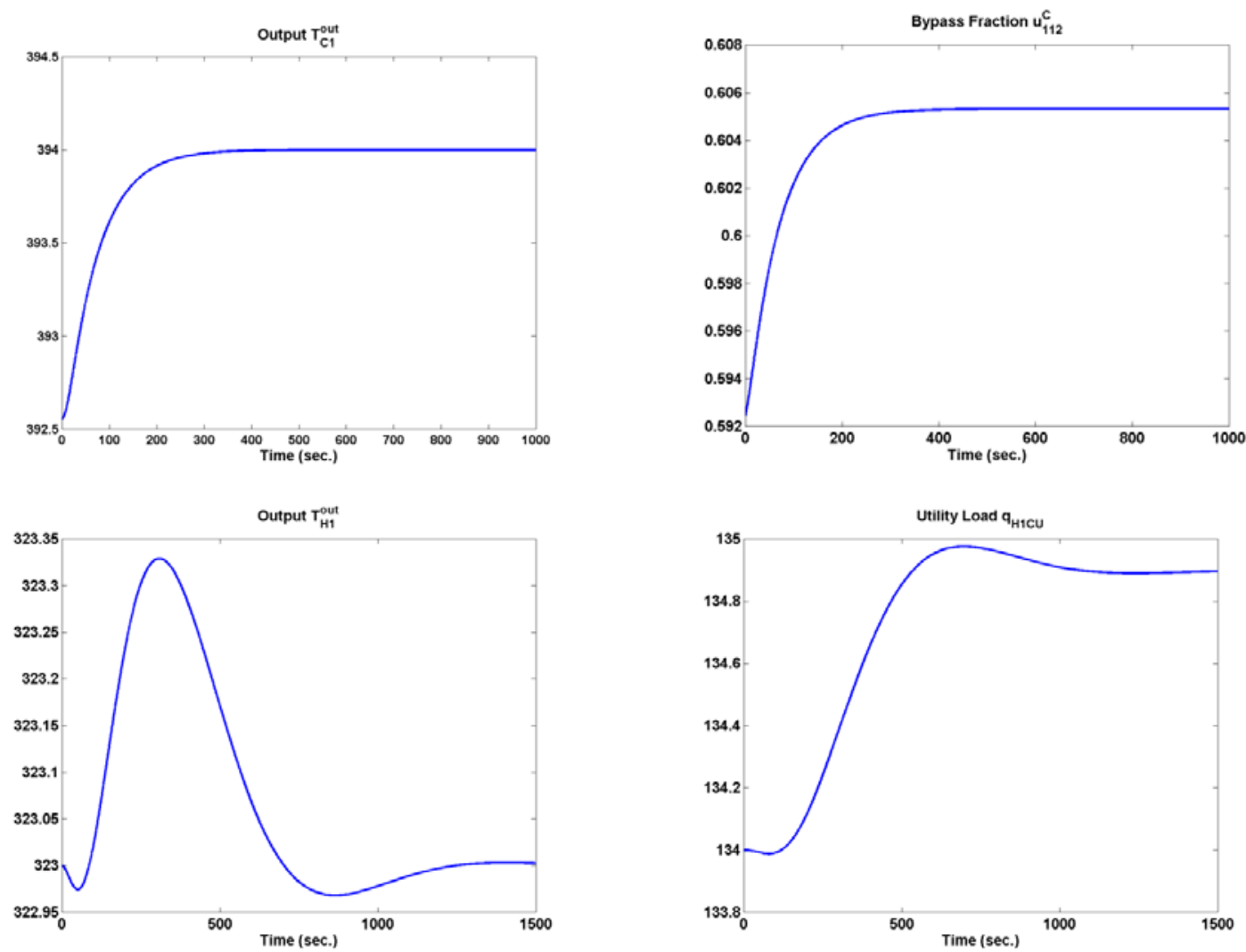

Figure C.3. Servo response for control loop 3, and effect on loop 1 - example 1. 

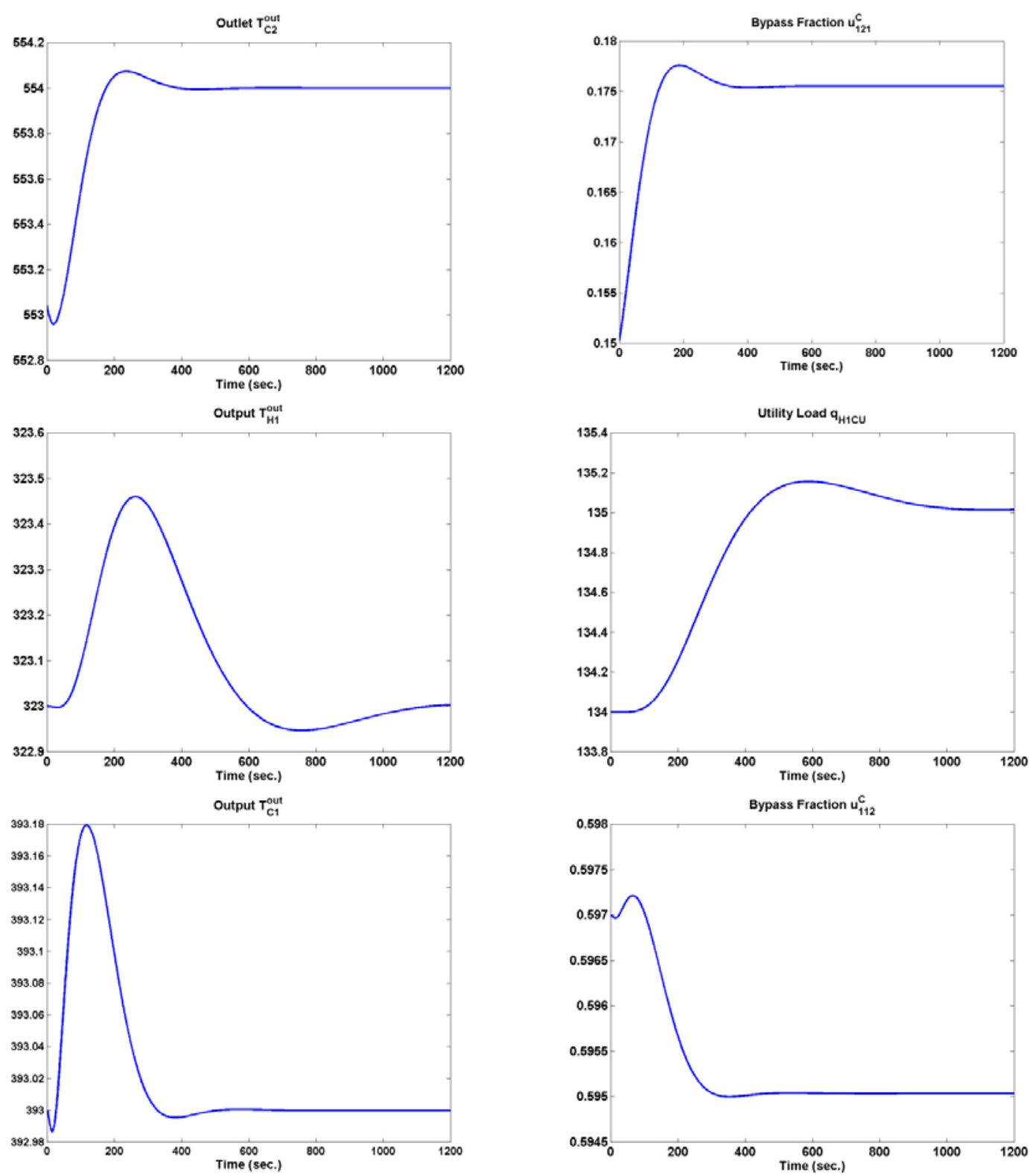

Figure C.4. Servo response for control loop 4, and effect on loop 1 and 3- example 1.
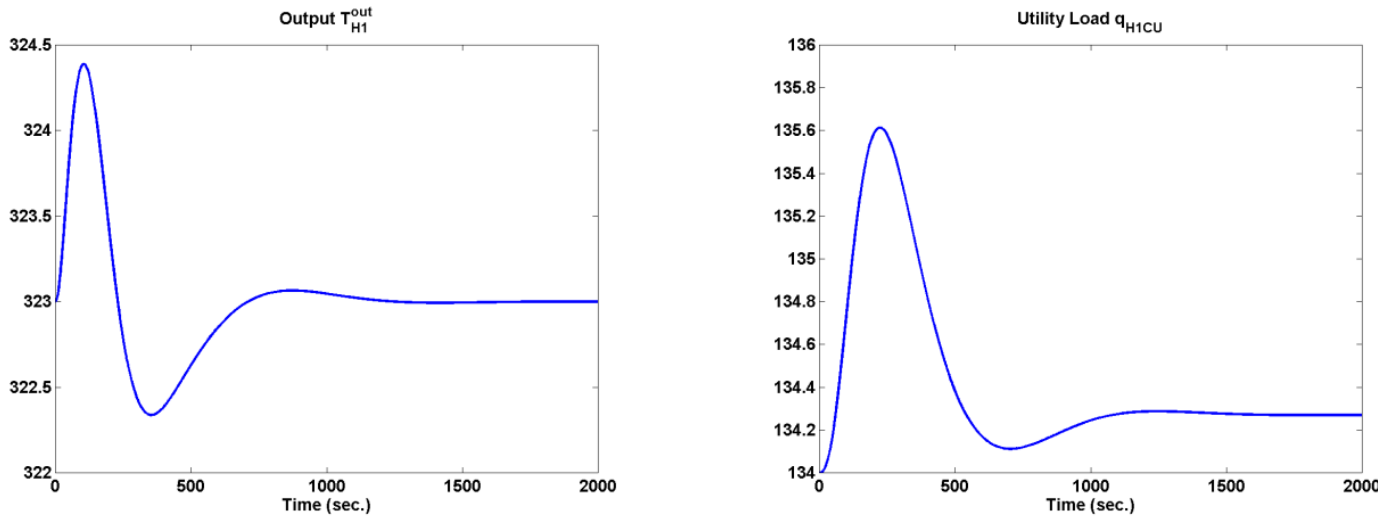

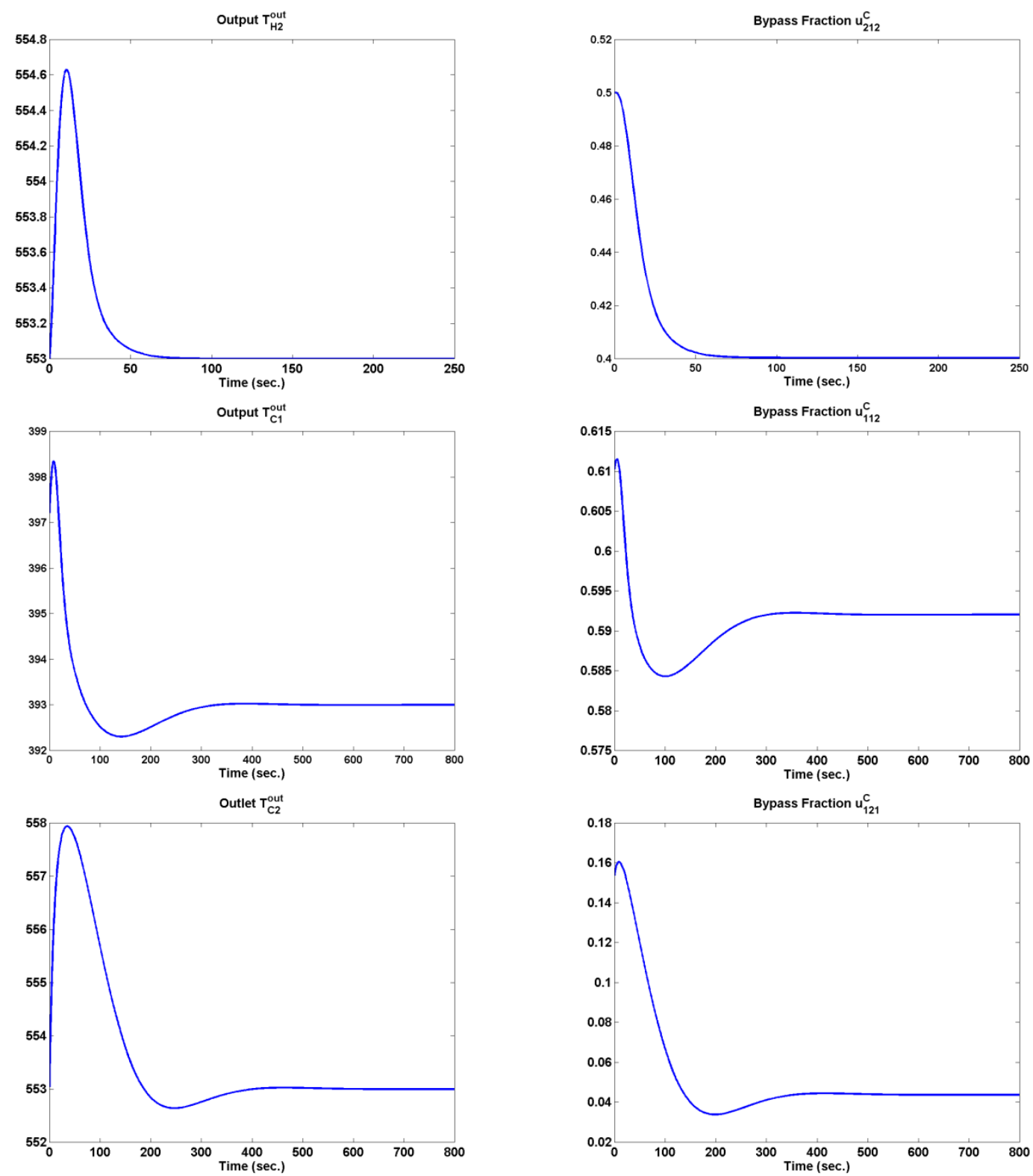

Figure C.5. Regulatory response for all control loops - example 1.
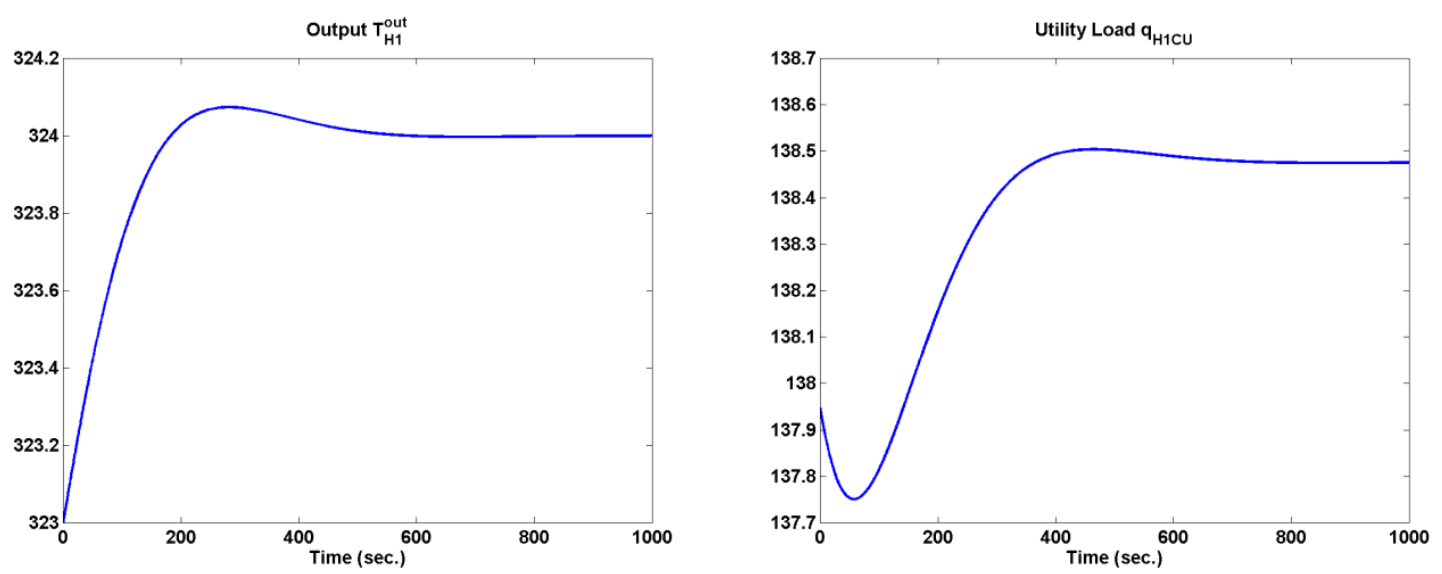

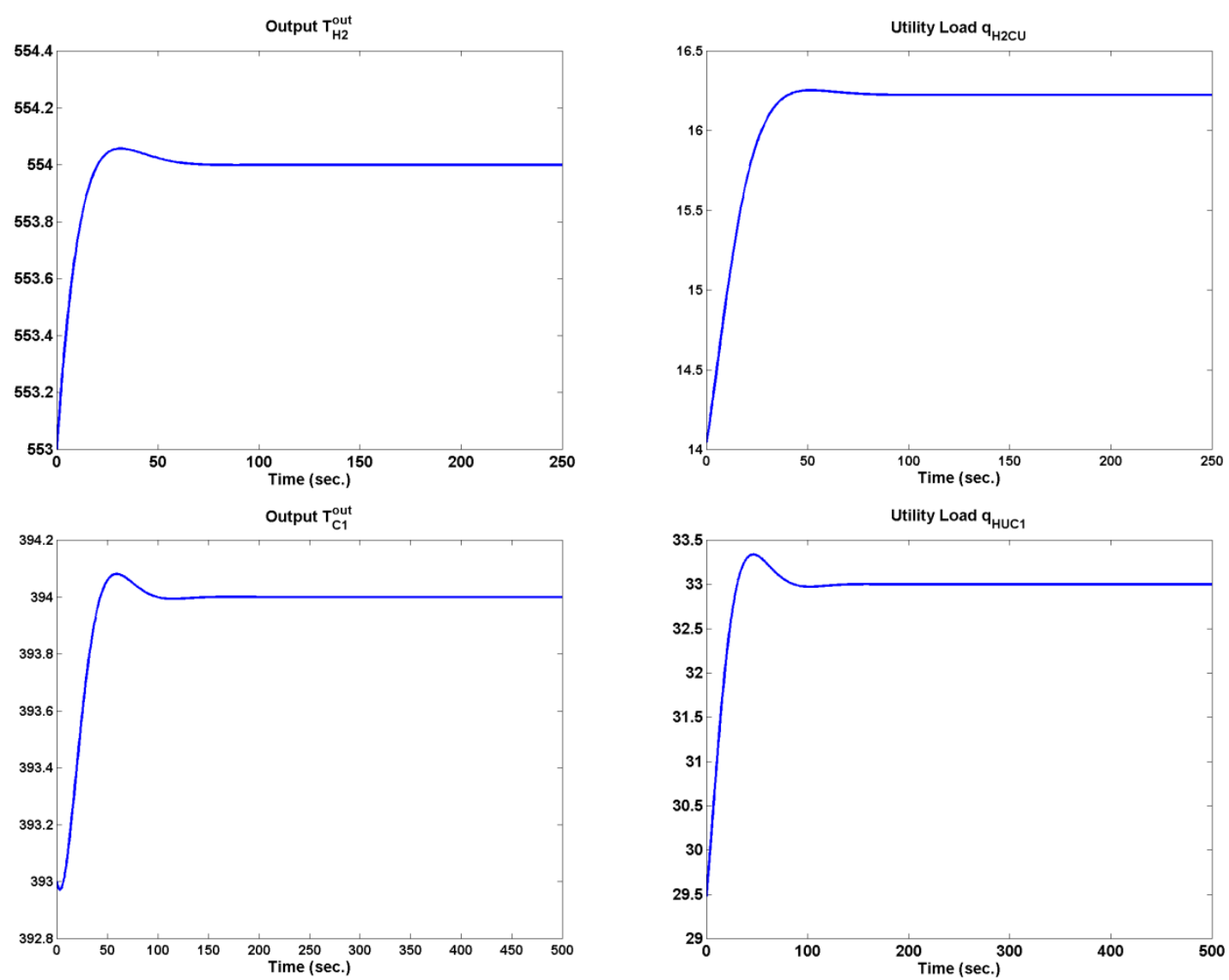

Figure C.6. Servo response for the loops controlled by utility loads - example 2.
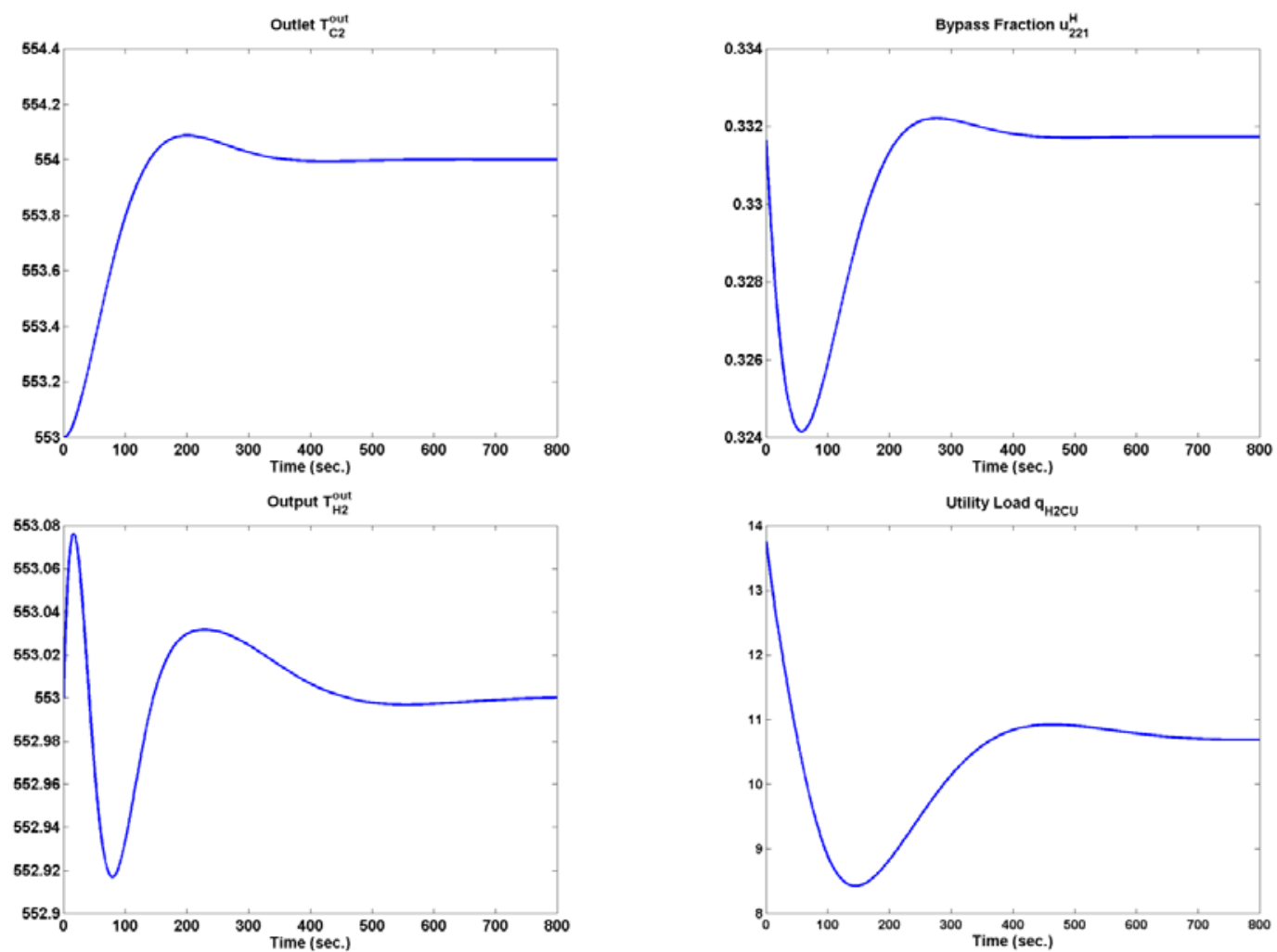

Figure C.7. Servo response for control loop 4 and effect on control loop 2 - example 2. 

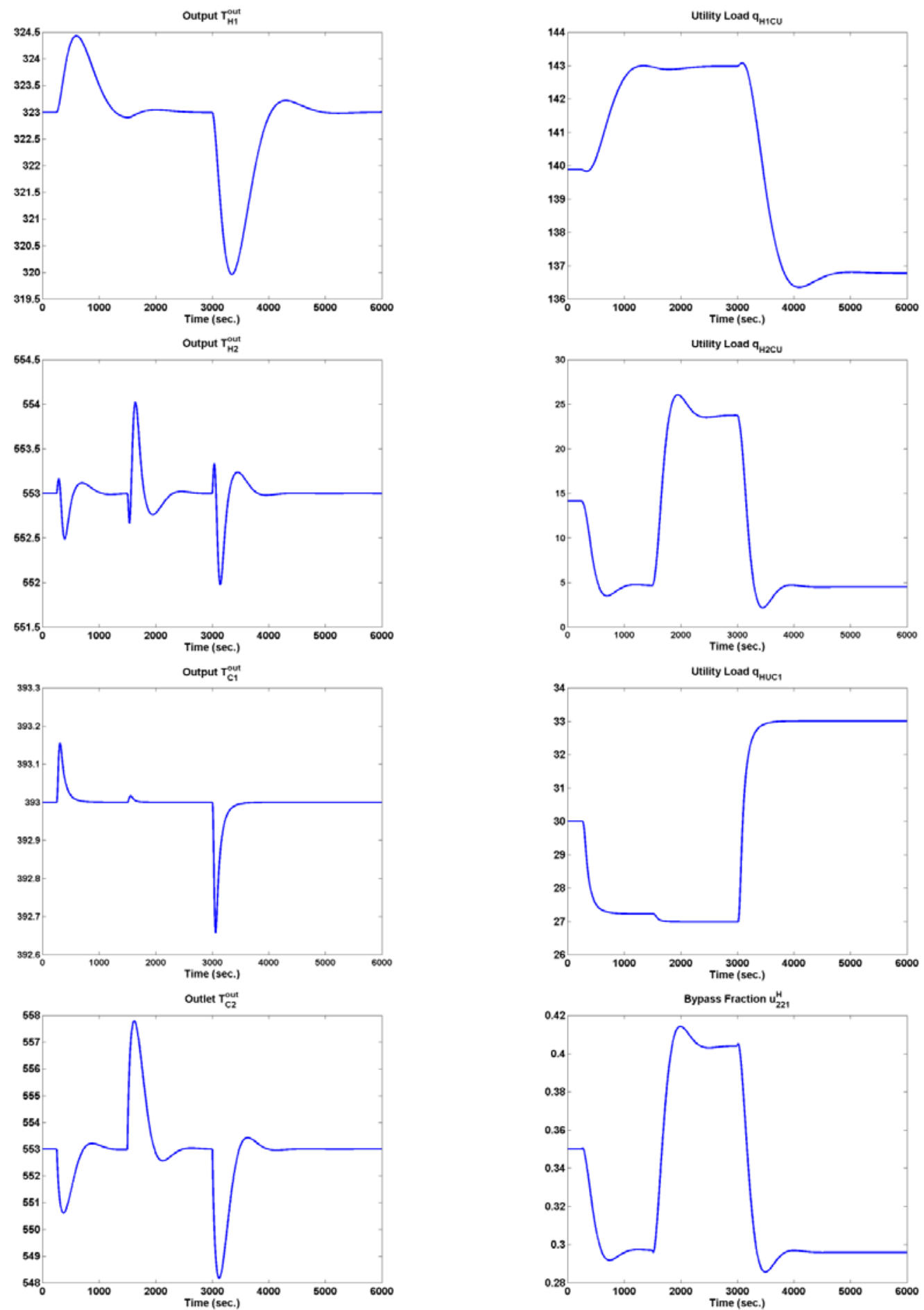

Figure C.8. Regulatory response for all control loops -example 2.
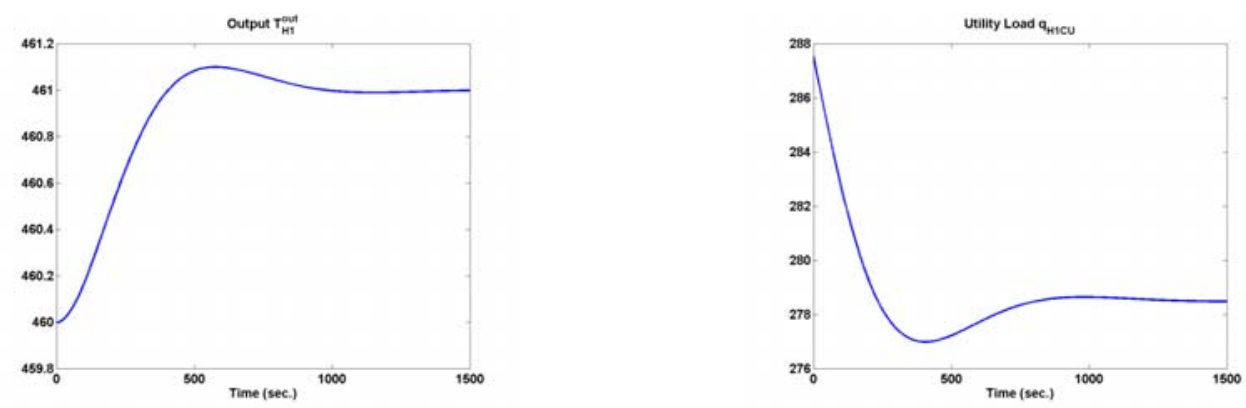

Figure C.9. Servo response for control loop 1 - example 3. 

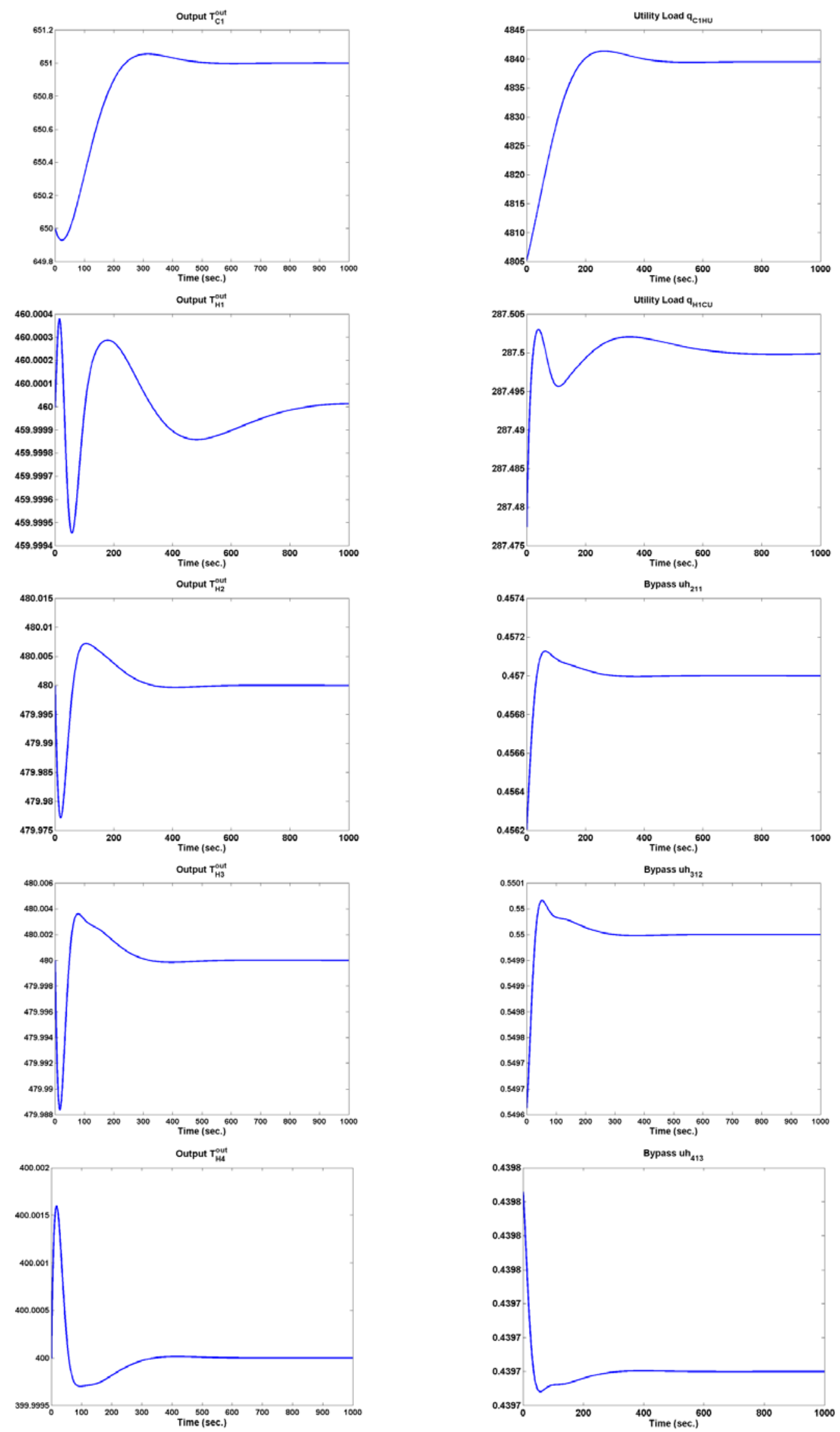

Figure C.10. Servo response for control loop 7 and effect on control loop 1,2,3 and 4. 

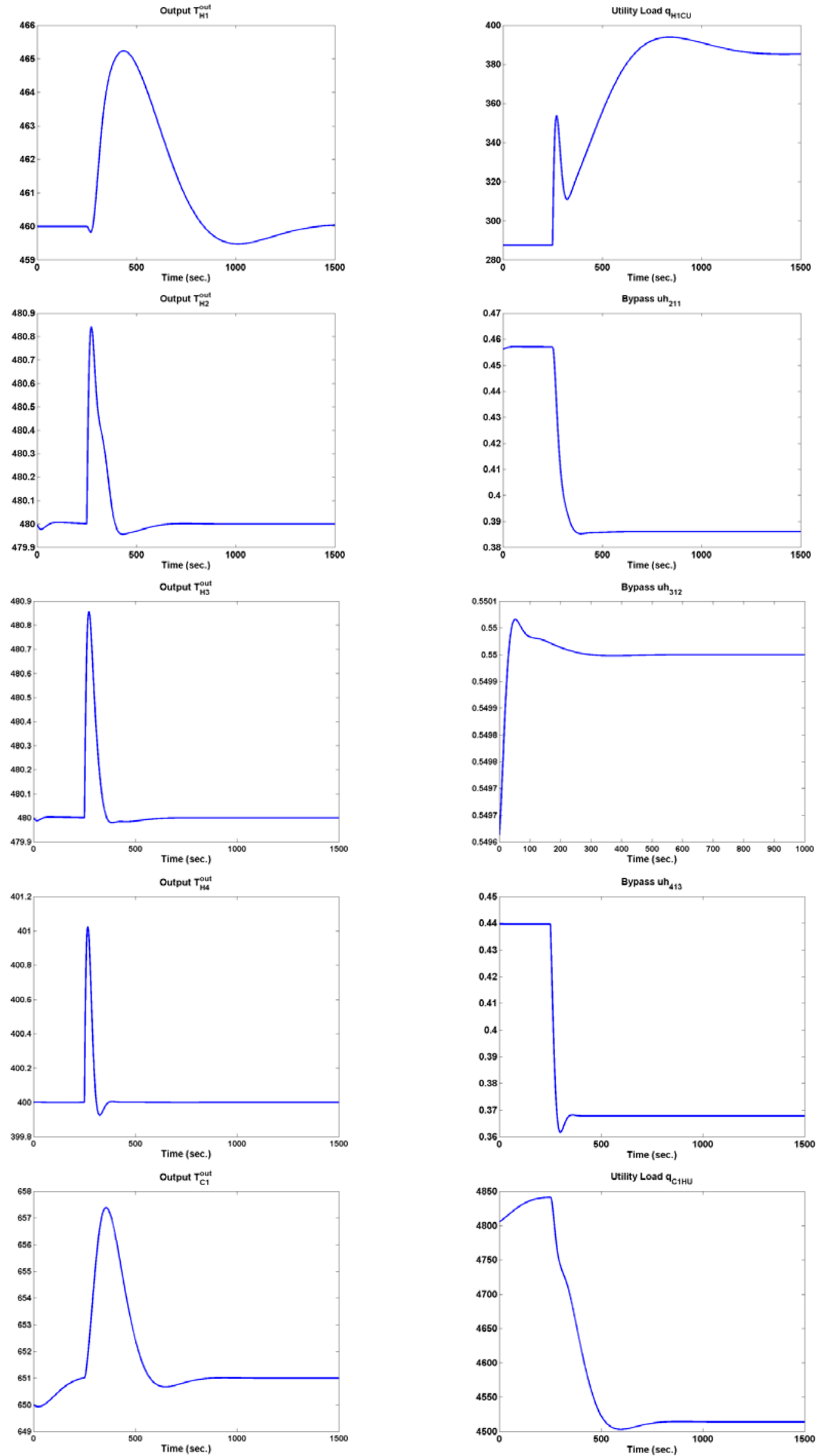

Figure C.11. Regulatory response for all control loops - example 3. 